\title{
Update on neutrino mixing in the early Universe
}

\author{
P. Di Bari \\ Deutsches Elektronen-Synchrotron DESY, 22603 Hamburg, Germany \\ (dibari@mail.desy.de)
}

\begin{abstract}
From the current cosmological observations of CMB and nuclear abundances we show, with an analytic procedure, that the total effective number of extra neutrino species $\Delta N_{\nu}^{\text {tot }}<0.3$. We also describe the possible signatures of non standard effects that could be revealed in future CMB observations. This cosmological information is then applied to neutrino mixing models. Taking into account the recent results from the SNO and SuperKamiokande experiments, disfavouring pure active to sterile neutrino oscillations, we show that all 4 neutrino mixing models, both of $2+2$ and $3+1$ type, lead to a full thermalization of the sterile neutrino flavor. Moreover such a sterile neutrino production excludes the possibility of an electron neutrino asymmetry generation and we conclude that $\Delta N_{\nu}^{\text {tot }} \simeq 1$, in disagreement with the cosmological bound. This result is valid under the assumption that the initial neutrino asymmetries are small. We suggest the existence of a second sterile neutrino flavor, with mixing properties such to generate a large electron neutrino asymmetry, as a possible way out.
\end{abstract}




\section{INTRODUCTION}

Neutrino mixing is the simplest explanation of the data from the atmospheric [四] and solar neutrino experiments [2,3], while alternative mechanisms are becoming more and more unlikely 4 . The results from the LSND experiment can be also explained by neutrino mixing [5].

In this work we investigate the possible cosmological effects of neutrino mixing. The theory of big bang nucleosynthesis has been proposed since long time as a probe for particle physics [6.7], but the systematic uncertainties in the measurements of nuclear abundances represent an obstacle for improvements. In the last two years different experiments confirmed the existence of acoustic peaks in the power spectrum of CMB temperature anisotropies [8], from which it has been possible to measure, with improved precision, many different cosmological parameters [9 12]. The precision of these measurements will be further improved by the new satellite experiments: the MAP satellite, already launched and on the way to its final orbit about the L2 Lagrangian point [13] and the PLANCK satellite whose launch is scheduled in the year 2007 [14]. These new observations represent a way to integrate the nuclear abundances observations partly overcoming the obstacle of systematic uncertainties and thus offering new opportunities to detect or constraint new physics in the early Universe, in particular the effects of neutrino mixing.

In the section II we describe a simple new analytic and graphical procedure to confront a large class of possible non standard effects with the cosmological observations. We consider both the present situation, finding that the total effective number of neutrino species $\Delta N_{\nu}^{\text {tot }}<0.3$, but we also point out which results from future observations could be interpreted as signatures of non standard effects. In sections III-IV-IV we examine the specific predictions of those neutrino mixing models that have been proposed to explain the current data, including or excluding the LSND experiment and, with the new procedure, we confront them with the cosmological observations. We explain why the early Universe encounters difficulties in detecting effects from three ordinary neutrino mixing models (section III), while it is emphasized the unique capability of the early Universe to probe a mixing with a new sterile neutrino sector even for very small mixing angles, otherwise out of the reach of Earth experiments (section IV). In the case of four neutrino mixing models (section V) we study the cosmological predictions using the results obtained in the simple active-sterile neutrino mixing and neglecting the possible presence of phases. We find the remarkable results that the new data from the SNO and SK experiments favour those four neutrino mixing models, both of ' $2+2$ ' and ' $3+1$ type', that lead to a final $\Delta N_{\nu}^{\text {tot }} \simeq 1$. In section VI we show how an additional sterile neutrino flavor could solve the disagreement with the cosmological bound if its mixing is able to generate a large electron neutrino asymmetry generation that produces a negative contribution to $\Delta N_{\nu}^{\text {tot }}$. In section VII we conclude summarizing which are the possible signatures of neutrino mixing models that should be searched in future observation.

\section{COSMOLOGICAL OBSERVATIONS}




\section{A. Current constraints}

The recent observations of CMB anisotropies 10 12 provide a useful consistency test for the other cosmological observations. The interpretation of data depends on theoretical assumptions. Therefore, it is important that the simplest model used to fit the data, that makes use of 7 independent parameters, gives results that are consistent with the other observations. A combined analysis of the experiments allowing also for the presence of tensor fluctuations and a hot dark matter component by increasing the number of parameters to 11 [15], does not show hints of the presence of such components and is remarkable that, when information from galaxy clustering is added, an upper bound of $4.4 \mathrm{eV}$ on the sum of neutrino masses is found. Although these analysis support a cosmological consistency, one has to be aware that maybe we are excluding important possible effects that are still compatible with the data or that maybe some cosmological observations are affected by systematic uncertainties and are misleading us to wrong conclusions and to exclude important pieces of the picture. We will take the attitude to consider the simplest results as reasonable but at the same time we will check whether these assumptions are compatible with the neutrino mixing models that we will examine.

In this section, we attempt to quantify the possibility that some non standard effects of BBN arise from neutrino mixing models. In order to test neutrino mixing models, CMB anisotropies are particularly important because they provide a measurement of the baryon content. This information has an important role in constraining the presence of new physics when is taken into account in models of Big Bang Nucleosynthesys (BBN). The BOOMERanG and DASI collaborations find an identical value [10,11] 1:

$$
\left(\Omega_{b} h^{2}\right)^{C M B}=0.022_{-0.003}^{+0.004}
$$

while the MAXIMA collaboration finds $\left(\Omega_{b} h^{2}\right)^{C M B}=0.0325_{-0.0125}^{+0.0125}$ at $95 \%$ c.l. [12]. A combined analysis has been performed in [15] in which both hot dark matter and tensor fluctuations are allowed and it gives for the CMB alone at $95 \%$ c.l. $\left(\Omega_{b} h^{2}\right)^{C M B}=0.02_{-0.01}^{+0.06}$. If information from the IRAS PSCz survey on galaxy clustering is used then they find, at $68 \%$ c.l., $\left(\Omega_{b} h^{2}\right)^{C M B}=0.020_{-0.003}^{+0.003}$ [16]. This result practically coincides with (1), even though different assumptions have been used. Therefore this seems quite a stable and reasonable value to be used for our analysis.

The standard BBN model (SBBN) assumes the particle physics content of the standard model of particle physics (in particular zero masses and no mixing for neutrinos). Moreover it assumes that the neutrino distributions are described by the Fermi-Dirac ones with zero chemical potentials and with a temperature $T_{\nu} \propto R^{-1}$ [17]. The predicted primordial nuclear abundances are functions of the only parameter $\eta$, the baryon to photon ratio, related to $\Omega_{b} h^{2}$ by the simple relation $\eta_{10} \equiv 10^{10} \eta \simeq 273.6 \Omega_{b} h^{2}$. The value (四) for $\left(\Omega_{b} h^{2}\right)^{C M B}$

\footnotetext{
${ }^{1}$ We indicate $1 \sigma$ errors for all quantities unless differently explicitly indicated. More precisely the DASI experiment quotes at $68 \%$ c.l $\left(\Omega_{b} h^{2}\right)^{C M B}=0.022_{-0.033}^{+0.035}$.
} 
corresponds to have [2:

$$
\eta^{C M B}=6.0_{-0.8}^{+1.1}
$$

These predictions have to be compared with the measured values. A first group finds 'high' values for the primordial Helium abundance [18]:

$$
Y_{p}^{\exp }=0.244 \pm 0.002
$$

while a second group finds 'low' values 19]:

$$
Y_{p}^{\exp }=0.234 \pm 0.003
$$

At the moment there is a tendency to admit that there are systematic uncertainties in this kind of measurements and to unify the two ranges of values. We however prefer to continue to distinguish the two different measurements.

The primordial Deuterium abundance is measured in Quasars absorption systems at high redshift. This kind of measurements gives the result [20]:

$$
(D / H)^{\exp }=(3.0 \pm 0.4) \times 10^{-5}
$$

We will not consider measurements of the primordial Lithium abundance since it is not fully understood whether we are really able to estimate how stellar processes could have modified it to the present. A test for the SBBN means to check whether the following conditions are fullfilled:

$$
\begin{gathered}
Y_{p}^{S B B N}\left(\eta^{C M B}\right)=Y_{p}^{\exp } \\
(D / H)^{S B B N}\left(\eta^{C M B}\right)=(D / H)^{\exp }
\end{gathered}
$$

The functions $Y_{p}^{S B B N}(\eta)$ and $(D / H)^{S B B N}(\eta)$ do not have exact analytical expression but fits around $\eta=5$ give the results [21 23] [5:

$$
\begin{gathered}
Y_{p}^{S B B N}(\eta) \simeq 0.2466+0.01 \ln (\eta / 5) \\
(D / H)^{S B B N}(\eta) \simeq 3.6 \cdot 10^{-5}(\eta / 5)^{-1.6}
\end{gathered}
$$

Using the CMB value (2) for $\eta$, one finds that the SBBN predicts:

\footnotetext{
${ }^{2}$ From this moment we will always show values of $\eta$ in units of $10^{-10}$, omitting the subscript ' $10^{\text {' }}$ to simplify the notation.

${ }^{3}$ We are considering the neutrino heating from $e^{+}-e^{-}$annihilations as a non standard effect (see discussion later on) and thus we are subtracting this contribution $\left(\Delta Y_{p} \simeq 1.4 \times 10^{-4}\right.$ [24]) from the result found in [21]: $Y_{p}^{S B B N}\left(\eta=5, \tau_{n}=887 \mathrm{sec}\right)=0.2467$, where $\tau_{n}$ is the neutron life-time.
} 


$$
\begin{gathered}
Y_{p}^{S B B N}\left(\eta^{C M B}\right)=0.2484_{-0.0014}^{+0.0017} \\
(D / H)^{S B B N}\left(\eta^{C M B}\right)=(2.7 \pm 0.7) \times 10^{-5}
\end{gathered}
$$

If we compare these values with the experimental measurements we see that the $\mathrm{SBBN}$ is in agreement with the observations if high values of $Y_{p}$ are used, otherwise for low values of $Y_{p}$ there is a $4 \sigma$ discrepancy. Such a comparison of SBBN with the observations can be also done saying that SBBN predicts, from the current $Y_{p}^{\exp }$ and $(D / H)^{\exp }$, the following values for $\eta(3 \sigma)$ :

$$
\begin{aligned}
\eta_{\text {highY }_{\mathrm{p}}}^{\mathrm{SBBN}} & =3.8_{-1.8}^{+3.2} \\
\eta_{\text {lowY }_{\mathrm{p}}}^{\mathrm{SBBN}} & =1.4_{-0.8}^{+2.1} \\
\eta_{(D / H)}^{S B B N} & =5.6_{-1.1}^{+2.1}
\end{aligned}
$$

and comparing them with $\eta^{C M B}$ the same previous conclusions follow.

We want now to quantify the possibility that $\mathrm{BBN}$ is non standard and of course, in doing this, we will be particularly interested in those non standard BBN models that can result from neutrino mixing. In this case the possible non standard effects are of two kinds and quite well known. The first effect is the possibility that the number of energy density degrees of freedom $g_{\rho} \equiv\left(30 / \pi^{2}\right)\left(\rho / T^{4}\right)$ differs from its SBBN value $g_{\rho}^{S B B N}=(22 / 4)+(21 / 4)\left(T_{\nu} / T\right)^{4}$ before or during the $\mathrm{BBN}$ period. In this way the expansion rate and the standard $\mathrm{BBN}$ predictions for the primordial nuclear abundances would be modified [6]. The change of $g_{\rho}$ can be expressed in terms of the (effective) extra number of neutrino species $[7] \Delta N_{\nu}^{\rho}$ :

$$
g_{\rho}=g_{\rho}^{S B B N}+\frac{7}{4} \Delta N_{\nu}^{\rho}\left(\frac{T_{\nu}}{T}\right)^{4}
$$

From the definition of $g_{\rho}$ it follows that $\Delta N_{\nu}^{\rho}$ is related to the neutrino energy densities by the following simple expression:

$$
\Delta N_{\nu}^{\rho}=\sum_{X} \frac{\rho_{X}+\rho_{\bar{X}}}{\rho_{0}}-3
$$

where $\rho_{0}=\left(7 \pi^{2} / 120\right) T_{\nu}^{4}$ and the ' $\mathrm{X}$ '-particles include the three ordinary neutrinos plus possible new species (we will be interested to possible new sterile neutrino flavors). Again we can make use of linear fits that account for the contribution of a non zero $\Delta N_{\nu}^{\rho}$ in the $\mathrm{BBN}$ predictions for the primordial nuclear abundances t:

$$
Y_{p}^{B B N}\left(\eta, \Delta N_{\nu}^{\rho}\right) \simeq Y_{p}^{S B B N}(\eta)+0.0137 \Delta N_{\nu}^{\rho}
$$

\footnotetext{
${ }^{4}$ The number 0.0137 can be inferred from the expansion given in 21] for $\eta \simeq 6$. While the dependence of $(D / H)$ on $\Delta N_{\nu}^{\rho}$ can be easily calculated considering that this abundance stays constant for $\eta / \sqrt{g_{\rho}}=$ const 25$]$.
} 


$$
(D / H)^{B B N}\left(\eta, \Delta N_{\nu}^{\rho}\right) \simeq(D / H)^{S B B N}(\eta)\left(1+0.135 \Delta N_{\nu}^{\rho}\right)^{0.8}
$$

A second class of deviations from $S B B N$ are those related to distortions of electron neutrino and anti-neutrino SBBN distributions, given by the Fermi-Dirac distribution with zero chemical potential (the same for neutrinos and anti-neutrinos). In general deviations cannot be described in terms of a finite number set of parameters but by an infinite number of parameters (the occupation numbers for each quantum state with a given momentum). However one can first calculate the change in $Y_{p}$ caused by these deviations and then normalize this change by introducing the quantity:

$$
\Delta N_{\nu}^{f_{\nu_{e}}} \equiv \frac{\left[Y_{p}\left(\eta, \Delta N_{\nu}^{\rho}, \delta f_{\nu_{e}, \bar{\nu}_{e}}\right)-Y_{p}\left(\eta, \Delta N_{\nu}^{\rho}\right)\right]}{0.0137}
$$

In this way one weighs the effect of distortions in terms of the presence of extra number neutrino species. A specific model of non standard BBN should be able to specify the deviations $\delta f_{\nu_{e}, \bar{\nu}_{e}}(p, t)$ at each momentum and during all the period of BBN. However it has to be remarked that once that the neutron to proton ratio has frozen, the electron neutrino ditributions do not have any more a direct role in the nuclear reactions. Thus everything will depend only on the frozen value of $n / p$ and still on $\Delta N_{\nu}^{\rho}$. This means that the Deuterium abundance will depend only indirectly on the electron neutrino distortions through the quantity $\Delta N_{\nu}^{f_{\nu}}$. Actually such a dependence is very weak and we will neglect it. Of course different $\delta f_{\nu_{e}, \bar{\nu}_{e}}$ can produce the same $\Delta N_{\nu}^{f_{\nu e}}$ and this degeneracy represents a lost of information f. The predictions of such non standard BBN models can again be compared with the experimental observations:

$$
\begin{gathered}
Y_{p}^{B B N}\left(\eta^{C M B}, \Delta N_{\nu}^{\rho}, \delta f_{\nu_{e}, \bar{\nu}_{e}}\right)=Y_{p}^{\exp } \\
(D / H)^{B B N}\left(\eta^{C M B}, \Delta N_{\nu}^{\rho}\right)=(D / H)^{\exp }
\end{gathered}
$$

The $Y_{p}$ measurement puts constraint on the quantity

\footnotetext{
${ }^{5}$ The only way to have more information on the $\delta f_{\nu_{e}, \bar{\nu}_{e}}$ would be to detect the electron relic neutrino distributions from which one could infer their values during BBN. Unfortunately relic neutrinos detection appears at the moment beyond the current observations but there are some interesting developments from the study of $\mathrm{UHE} \nu$ scattering on relic neutrinos and producing $\mathrm{Z}$ bursts [26].

${ }^{6}$ Note there could be other kinds of non standard effects non considered here, like those ones associated with the possibility that during the BBN epoch there were baryon inhomogeneities on the scale of neutron diffusion length (see [27] and references therein). The quantity $\Delta N_{\nu}^{\text {tot }}-\Delta N_{\nu}^{\rho}$ would assume a more general interpretation and depend also on other non standard parameters like the size of inhomogenenities and thus should be more generally indicated for example with $\Delta N_{\nu}^{f_{\nu}+\mathrm{inh}}$. In this paper we are interested to focus on non standard effects from neutrino mixing models and thus we completely neglect the possibility for these kinds of inhomogeneities but it is interesting that this procedure could be employed also in a different context.
} 


$$
\Delta N_{\nu}^{\mathrm{tot}}=\Delta N_{\nu}^{\rho}+\Delta N_{\nu}^{f_{\nu_{e}}} \equiv \Delta Y_{p}^{B B N} / 0.0137
$$

At $3 \sigma$, assuming high values for $Y_{p}^{\exp }$, we find :

$$
\Delta N_{\nu}^{\text {tot }}=-0.3_{-0.6}^{+0.6}
$$

while assuming low values we find:

$$
\Delta N_{\nu}^{\text {tot }}=-1.05 \pm 0.75
$$

The Deuterium abundance provides a complementary information on $\Delta N_{\nu}^{\rho}$ and the comparison between the prediction and the observed value gives, conservatively at a $3 \sigma$ level, an upper bound on $\Delta N_{\nu}^{\rho}$ :

$$
\left(\Delta N_{\nu}^{\rho}\right)^{B B N} \lesssim 13
$$

while a lower bound is still not obtained with the current precision of measurements. The constraints (23), (24) and (25) are shown in figure 1, in a plot $\Delta N_{\nu}^{\text {tot }}-\Delta N_{\nu}^{\rho}$.

\section{B. Future observations and possible signatures}

It is interesting to see how one can expect that these constraints will improve from future CMB measurements of $\eta$. The Planck experiment should be able to measure $\eta$ with a precision at the level of $1 \%$ or less [28]. In this way the uncertainties on the theoretical predictions of the nuclear abundances, $Y_{p}^{B B N}$ and $(D / H)^{B B N}$, will become negligible compared to the errors on the experimental values.

Assuming that the future measured value $\eta^{C M B}$ will correspond to the current central value of $(D / H)$ in SBBN, $\eta=5.6$ (see Eq.(14)), then the current Deuterium observations will constraint $\Delta N_{\nu}^{\rho}$ to be $\lesssim 4.0(3 \sigma)$ (the horizontal thick dashed line in figure 1) while still one does not get a lower bound ?. The same exercise can be performed with $Y_{p}$ to see how the constraints on $\Delta N_{\nu}^{\text {tot }}$ would improve and the result is shown with vertical dashed lines in figure 1. This time the improvement is slight because the $Y_{p}$ abundance is much less sensitive to $\eta$ than $(D / H)$ and the error on $\Delta N_{\nu}^{\text {tot }}$ is dominated by the error on $Y_{p}^{\exp }$. The two constraints together, from $(D / H)$ and $Y_{p}$, give the gray region in figure 1 with thick dashed line contours.

Differently it could happen that future CMB observations will indicate $\eta^{C M B}>\eta_{\text {highY }}^{S B B N}$ (see Eq. (12)). In figure 1 it is shown, in light gray, the allowed cosmological region (at

\footnotetext{
${ }^{7}$ One finds $\Delta N_{\nu}^{\rho} \gtrsim-4$ that is not particularly meaningful. At $2 \sigma$ one gets $\Delta N_{\nu}^{\rho} \gtrsim-2$, that implies the presence of at least one standard neutrino species. Note that a model in which a $\mathrm{MeV} \tau$ neutrino was decaying prior the onset of BBN was proposed to solve the BBN crisis from low $Y_{p}^{\exp }$ values [29]. In such a case one can get negative values of $\Delta N_{\nu}^{\rho}$ as low as -1 . Nowaday such a model is very disfavored by the neutrino oscillations experiments but nevertheless it gives an example of why it is not meaningless to put a negative lower bound on $\Delta N_{\nu}^{\rho}$, that moreover can be considered a sort of consistency check of the basic BBN assumptions.
} 
$3 \sigma)$ in the plane $\Delta N_{\nu}^{\text {tot }}-\Delta N_{\nu}^{\rho}$ for $\eta^{C M B}=7.0$ (1\% error) for a 'low +high' joint range of $Y_{p}$ values. The SBBN would be ruled out at $3 \sigma$ and negative $\Delta N_{\nu}^{\text {tot }}<\Delta N_{\nu}^{\rho}$ would be required. Therefore, in future, a $1 \%$ error measurement $\eta^{C M B} \gtrsim 7$ (or $\left(\Omega_{b} h^{2}\right)^{C M B} \gtrsim 0.0256$ ), will represent the opportunity to have a significant signature of non standard BBN effects with the current nuclear abundances observations. On the other hand the current allowed $3 \sigma$ range of values of $\eta^{C M B}, 3.6-9.9$ (see Eq. (2)), excludes already now the possibility that a future $1 \%$ error measurement of $\eta^{C M B}$, with current $Y_{p}$ measurements, can give indications for positive values of $\Delta N_{\nu}^{f_{\nu e}}$, that means $\Delta N_{\nu}^{\text {tot }}<\Delta N_{\nu}^{\rho}$, because it would require $\eta^{C M B} \lesssim 2$ using high $Y_{p}$ values (see Eq. 12) and even lower values of $\eta^{C M B}$ using low $Y_{p}$ values.

From the Eq. (14), one can see that from a $1 \%$ error measurement $\eta^{C M B} \gtrsim 7.7$ the Deuterium abundance would require also $\Delta N_{\nu}^{\rho}>0$ (other than negative $\Delta N_{\nu}^{f_{\nu}}$ ). On the converse, for $\eta^{C M B} \lesssim 4.5$, negative values of $\Delta N_{\nu}^{\rho}$ would be required.

Another important improvement, from future observations of CMB anisotropies, will be the direct measurement of $\Delta N_{\nu}^{\rho}$. The presence of an extra radiative component changes the CMB spectrum, in particular leading to the enhancement of the height of first acoustic peak. At the present, a completely independent measurement of $\Delta N_{\nu}^{\rho}$ from CMB anisotropies gives a very loose constraint $\left(\Delta N_{\nu}^{\rho}\right)^{C M B}<19$ (at $95 \%$ c.l., CMB alone) [30]. However future MAP and Planck satellite experiments should reach a precision of $10^{-3}-10^{-1}$ according to whether the information on the other cosmological parameters from other observations will be used or not and whether the CMB polarization will be measured or not [31]. In figure 1 we indicated with horizontal thin dashed lines a realistic future constraint $\left|\left(\Delta N_{\nu}^{\rho}\right)^{C M B}\right|<0.1$. It has to be said that the CMB observations will measure $\Delta N_{\nu}^{\rho}$ around the time of recombination and thus it could be in principle different from the value of $\Delta N_{\nu}^{\rho}$ during the earlier period of $\mathrm{BBN}$ if some intervening effect modified it. For example $\left(\Delta N_{\nu}^{\rho}\right)^{\mathrm{CMB}}$ can be higher of $\left(\Delta N_{\nu}^{\rho}\right)^{\mathrm{BBN}}$ in the case of massive neutrino decays. In this case a comparison between the two quantities will test the 'relativity parameter' $\alpha \propto m_{\nu}^{2} \tau$ [32].

Their comparison could give also another result: $\left(\Delta N_{\nu}^{\rho}\right)^{\mathrm{CMB}}<\left(\Delta N_{\nu}^{\rho}\right)^{\mathrm{BBN}}$. This is possible only if one can say that $\left(\Delta N_{\nu}^{\rho}\right)^{\mathrm{BBN}}>0$. If one looks at the expressions (8) and (14), such a conclusion is possible if future $1 \%$ error observations will give $\eta^{C M B} \gtrsim 7.7$. For example from $\eta^{C M B} \gtrsim 7.8$ one can deduce $\left(\Delta N_{\nu}^{\rho}\right)^{B B N} \gtrsim 0.2$, while it can happen at the same time that CMB constraints $\Delta N_{\nu}^{C M B} \lesssim 0.1$. This paradoxical situation could occur if $\Delta N_{\nu}^{\text {tot }}$ is inhomogenous. We neglected a dependence of $(D / H)^{B B N}$ on $\Delta N_{\nu}^{\text {tot }}$, that means on $Y_{p}$ or, equivalently, on the frozen value of neutron to proton ratio. This because the $Y_{p}$ observations suggest that $Y_{p}$ cannot differ from the SBBN value so much to modify $(D / H)$ in a sensible way, while the value of $\Delta N_{\nu}^{\rho}$ is much less constrained and it can considerably alter the value of $(D / H)$. However the observations measure $Y_{p}$ only within about $100 \mathrm{Mpc}$ around us, while the $(D / H)$ abundance is measured in the quasars absorption systems at much larger distances. Thus it cannot be excluded that $Y_{p}$ can be 'there' much larger than what we observe around us [33]. The amplitude of CMB anisotropies exclude the possibility that this spatial variation can be due to a inhomogenous $\Delta N_{\nu}^{\rho}$, and thus it can be due only to a inhomogeneous $\Delta N_{\nu}^{f_{\nu}}$ that should be 'there' much larger (and positive) than around us. In this case the $(D / H)$ nuclear abundance can be higher than what predicted by SBBN and compatible with $\eta^{C M B}>\eta^{S B B N}$. Such a possibility should be however accompanied by the observation of dispersion in the $(D / H)$ measurements in the range of values $(1.8-3.6) \times 10^{-5}$. 
Note that at the moment values of $\eta^{C M B} \gtrsim 7.7$ are already excluded at $1.5 \sigma$ and thus a small improvement in the measurement precision of $\eta^{C M B}$ should be able to disfavour (or reveal !) such a situation. However only constraining the dispersion in the values of measured $(D / H)$ can put more general limits on the presence of inhomogeneities in $\Delta N_{\nu}^{f_{\nu_{e}}}$.

Another important possibility is whether future observations will indicate $\Delta N_{\nu}^{\rho}>0.3$, because then, in order not to violate the bound $\Delta N_{\nu}^{\text {tot }}<0.3$, one can conclude that there is a negative contribution $\Delta N_{\nu}^{f_{\nu}}$.

\section{Two special cases of non standard effects}

The SBBN corresponds, in the plane $\Delta N_{\nu}^{\text {tot }}-\Delta N_{\nu}^{\rho}$, to the origin. One can consider the correction to the approximation of full neutrino decoupling at the time of electronpositron annihilations (implying $T_{\nu} \propto R^{-1}$ ). It has been shown that such a correction yields $\Delta N_{\nu}^{\text {tot }} \simeq 0.012$ and $\Delta N_{\nu}^{\rho} \simeq 0.034$ [24]. Thus the predictions of nuclear abundances within the standard model of particle physics do not exactly coincide with those of SBBN. In the optimistic case that future CMB observations will be able to detect $\Delta N_{\nu}^{\rho}$ as small as 0.01 , then the small effect of neutrino heating should be distinguished [31] and this would represent an important confirmation of the early Universe standard scenario below $\sim 10 \mathrm{MeV}$.

A particular subclass of non standard BBN models, of the type considered here, is that one in which neutrinos have still thermal distributions but with non zero chemical potentials (fulfilling the chemical equilibrium condition $\mu_{\bar{\nu}_{\alpha}}=-\mu_{\nu_{\alpha}}$ ). In this particular case one has the following correspondence:

$$
\Delta N_{\nu}^{\rho}=\sum_{\alpha}\left[\frac{30}{7}\left(\frac{\xi_{\alpha}}{\pi}\right)^{2}+\frac{15}{7}\left(\frac{\xi_{\alpha}}{\pi}\right)^{4}\right], \quad \Delta N_{\nu}^{f_{\nu e}} \simeq-16 \xi_{e}
$$

with $\xi_{\alpha} \equiv \mu_{\alpha} / T^{\natural}$. This kind of models have been largely studied in the literature since early times [17,34]. Constraints from the most recent cosmological observations have been also recently obtained in 35. Their procedure put constraints on $\Delta N_{\nu}^{\rho}$, assuming that is equal in $\mathrm{BBN}$ and $\mathrm{CMB}$ epochs, and on $\eta$ in a statistical combined way and taking into account a slight dependence of $\eta^{C M B}$ on $\Delta N_{\nu}^{\rho}$. This allows to get more restrictive constraints but in a more specific context and at the expenses of physical insight. In our procedure we get more conservative constraints becasue of the poorest statistical procedure. On the other hand we gain more physical insight from an analytical procedure valid in a more general framework in which we distinguish $\Delta N_{\nu}^{\rho}$ in $\mathrm{BBN}$ and $\mathrm{CMB}$, the role of $\Delta N_{\nu}^{f_{\nu e}}$ is emphasized as we need for our purposes, and we find a bound on $\Delta N_{\nu}^{\text {tot }}$ missing in [35. All these features are important for our following considerations.

\footnotetext{
${ }^{8}$ The second relation is a good approximation for $\left|\xi_{e}\right| \ll 1$ and $\Delta N_{\nu}^{\rho} \ll 20$.
} 


\section{THREE ORDINARY NEUTRINO MIXING}

With the exclusion of the LSND experiment, usually justified with the argument that it is the only experiment not yet confirmed by a second one, a three ordinary neutrino mixing can explain the current data from solar and atmospheric neutrino experiments. The three ordinary neutrino flavors eigenstates, $\left|\nu_{\alpha}\right\rangle(\alpha=e, \mu, \tau)$, are connected to three mass eigenstates $\left|\nu_{i}\right\rangle$ with definite masses $m_{i}(i=1,2,3)$, by a $3 \times 3$ neutrino mixing matrix $U$ :

$$
\left|\nu_{\alpha}\right\rangle=\sum_{i=1}^{3} U_{\alpha i}^{\star}\left|\nu_{i}\right\rangle
$$

The atmospheric neutrino experiments are then explained by the mixing of $\left|\nu_{2}\right\rangle$ and $\left|\nu_{3}\right\rangle$ mass eigenstates with $\left|m_{3}^{2}-m_{2}^{2}\right|=\delta m_{\mathrm{atm}}^{2} \simeq 2.5 \times 10^{-3} \mathrm{eV}^{2}$ with a large mixing angle $\sin ^{2} 2 \theta_{23} \gtrsim 0.88$ [1] and with a negligible $\left|U_{e 3}\right| \ll 1$ (as required by the CHOOZ experiment [36]) that implies a small mixing angle $\theta_{13}$. In this way the $\nu_{\mu}$ 's are converted almost only to $\nu_{\tau}$ 's. The solar neutrinos are explained by the mixing of $\nu_{1}$ and $\nu_{2}$ eigenstates with $\left|m_{2}^{2}-m_{1}^{2}\right|=\delta m_{\odot}^{2} \ll 10^{-3} \mathrm{eV}^{2}$. With the new data from the SNO experiment, large mixing angle solutions $\left(\sin ^{2} 2 \theta_{12} \simeq 1\right)$ are also favoured [37,38]. In this way the favoured three neutrino mixing models are those close to the the bimaximal mixing scenario [39]. A three ordinary neutrino mixing does not have relevant effects on the cosmological picture and in particular on the quantities $\Delta N_{\nu}^{\rho}$ and $\Delta N_{\nu}^{f_{\nu e}}$. It has been noted that a mixing of electron neutrinos with muon/tauon neutrinos during the period of freeze out of the neutron to proton ratio would exchange the abundances of the two types that are slightly different due to the different effect of neutrino heating [40]. In this way the effect of neutrino heating would change. However the neutrino heating effect is small like also the difference between the muon/tauon and the electron neutrino populations. Thus the bimaximal mixing would represent a correction of an already correcting effect. Therefore, at the moment, such a mixing model seems out of reach of cosmological investigation 1 .

\section{ACTIVE-STERILE NEUTRINO MIXING}

An explanation in terms of neutrino mixing of the solar and atmospheric neutrino experiments, together with the LSND experiment, implies three different scales of mass squared differences: $\delta m_{\odot}^{2} \ll \delta m_{\text {atm }}^{2} \ll \delta m_{\mathrm{LSND}}^{2}$. This requires the existence of at least one new neutrino flavor [41] that has to be sterile in order to escape the constraint $N_{\nu}^{Z}=3.00 \pm 0.06$ from the invisible decay width of the Z-boson [42]. A two neutrino mixing between one ordinary neutrino flavor $\nu_{\alpha}$ and one sterile neutrino flavor $\nu_{s}$ is the simplest case of mixing involving new sterile neutrino flavors. With the new data from the atmospheric and the solar neutrino experiments, such a simple scheme seems to be excluded, as the three ordinary

\footnotetext{
${ }^{9}$ Cosmology is however useful to get information on the mass pattern, in particular structure formation and CMB observations are currently sensitive to a few eV's masses [15], while the PLANCK experiment will be be sensitive to a few $0.1 \mathrm{eV}$ 's masses 28 .
} 
neutrino flavors appear to be mixed among themselves. However the solutions of the kinetic equations, necessary to calculate $\Delta N_{\nu}^{\rho}$ and $\Delta N_{\nu}^{\text {tot }}$, present many difficulties and this basic case represents an important starting point. Moreover it can represent a limit case for some of the possible sub-mixings within a realistic multiflavor mixing, as we will see in the next section. It is described by only two parameters, $\delta m^{2} \equiv m_{2}^{2}-m_{1}^{2}$ and $s^{2} \equiv \sin ^{2} 2 \theta_{0}$, where $\theta_{0}$ is the vacuum mixing angle. The $\left|\nu_{1}\right\rangle$ mass eigenstate $\left(\left|\nu_{2}\right\rangle\right)$ corresponds to the ordinary (sterile) neutrino eigenstate in the limit of no mixing. The straightest cosmological effect is the sterile neutrino production with a consequent generation of a $\Delta N_{\nu}^{\rho}$ that can be as high as 1 in the case of full thermalization. In doing these calculations one has to make some assumptions about the initial value of the ordinary neutrino asymmetries. We define the asymmetry of a lepton (baryon) particle species $X$ as:

$$
L_{X}\left(B_{X}\right) \equiv \frac{N_{X}-N_{\bar{X}}}{N_{\gamma}^{\text {in }}}
$$

with $N_{X}$ being the particle number per comoving volume and $N_{\gamma}^{\text {in }}$ is the number of photons per comoving volume at an initial temperature $T^{\text {in }} \simeq 10 \mathrm{MeV} \gg m_{\mathrm{el}} / 2 \simeq 0.25 \mathrm{MeV}$. The (effective) total $\alpha$-neutrino asymmetry is defined as:

$$
L^{(\alpha)} \equiv L_{\nu_{\alpha}}+L_{\nu_{e}}+L_{\nu_{\mu}}+L_{\nu_{\tau}}+Q_{\alpha}
$$

with $Q_{\mu, \tau}=-(1 / 2) B_{n}$ and $Q_{e}=L_{e}-(1 / 2) B_{n}$. For initial values $\left|L^{(\alpha)}\right| \ll$ $10^{-6}\left(\left|\delta m^{2}\right| / \mathrm{eV}^{2}\right)^{1 / 3}$ ('small' neutrino asymmetries) the effects on the oscillations can be neglected, while for much higher values an initial neutrino asymmetry can modify, usually suppressing, the sterile neutrino production prior the onset of BBN [43,44]. For small neutrino asymmetries, the sterile neutrino production is given by [45,44:

$$
N_{\nu_{s}}^{\rho}=1-\exp \left[-g_{ \pm}^{\alpha}\left(s^{2},\left|\delta m^{2}\right| / \mathrm{eV}^{2}\right)\right]
$$

The function $g_{ \pm}^{\alpha}\left(s^{2},\left|\delta m^{2}\right| / \mathrm{eV}^{2}\right)$ can be written in the form:

$$
g_{ \pm}^{\alpha}\left(s^{2}, \delta m^{2} / \mathrm{eV}^{2}\right)=K_{\alpha} F_{ \pm}^{\alpha}\left(s^{2}\right) s^{2} \sqrt{\left|\delta m^{2}\right| / \mathrm{eV}^{2}}
$$

where the subscript $+(-)$ stands for positive (negative) $\delta m^{2}$ and $K_{\alpha} \simeq 657$ (898) for $\alpha=$ $e(\mu, \tau)$. The function $F_{ \pm}^{\alpha}\left(s^{2}\right)$ is given by the following integral:

$$
F_{ \pm}^{\alpha}\left(s^{2}\right)=\int_{0}^{\infty} d t \frac{t^{2}}{s^{2}+d_{0}^{2} t^{12}+\left(c \pm t^{6}\right)^{2}}
$$

with $c \equiv \cos 2 \theta_{0}$ and $d_{0} \simeq 0.008$ (0.02) for $\alpha=e(\mu, \tau)$. These results have been obtained within the static approximation [46] that neglects the MSW effect at the resonance. In the resonant case, for negative $\delta m^{2}$, this approximation holds only for very small mixing angles $\left(s^{2} \ll 10^{-4}\right)$ and for small neutrino asymmetries [47]. Note that $\Delta N_{\nu}^{\rho}=N_{\nu_{s}}^{\rho}+$ $\left(\sum_{\beta}^{e, \mu, \tau} N_{\nu_{\beta}}^{\rho}-3\right)$, where the second term takes into account the depletion of ordinary neutrinos that is negligigible when the bulk of sterile neutrino production occurs before the neutrino chemical decoupling and in this case one has approximately $\Delta N_{\nu}^{\rho} \simeq N_{\nu_{s}}^{\rho}$. This is verified 
for $\left|\delta m^{2}\right| \gtrsim 10^{-4} \mathrm{eV}$ that, for values of $N_{\nu_{s}} \gtrsim 0.01$, corresponds to have small mixing angles $s^{2} \lesssim 10^{-2}$ in the non resonant case and $s^{2} \lesssim 10^{-4}$ in the resonant case. In these regimes of small mixing angles, the function $F_{ \pm}^{\alpha}\left(s^{2}\right)$ is well approximated by its asymptotic value $F_{ \pm}^{\alpha}(0)$ and one has for $\alpha=e(\mu, \tau)$ :

$$
\begin{aligned}
& g_{+}^{\alpha}\left(s^{2} \lesssim 10^{-2}, 1\right) / s^{2} \simeq K_{\alpha} F_{+}^{\alpha}(0) \simeq 1.69(2.33) 10^{2} \\
& g_{-}^{\alpha}\left(s^{2} \lesssim 10^{-4}, 1\right) / s^{2} \simeq K_{\alpha} F_{-}^{\alpha}(0) \simeq 4.28(2.27) 10^{4}
\end{aligned}
$$

In the non resonant case these analytical results agree very well with the numerical ones found in 48 四 for $s^{2} \geq 10^{-4}$, while in 49 it is claimed that $\Delta N_{\nu}^{\rho}$ is approximately three times lower.

All these results have been obtained assuming small neutrino asymmetries. However in the resonant case, at small mixing angles, even if one starts with small neutrino asymmetries, a large $\alpha$-neutrino asymmetry is generated around the critical temperature:

$$
T_{c} \simeq 15.0(18.6) \mathrm{MeV}\left(\left|\delta m^{2}\right| / \mathrm{eV}^{2}\right)^{1 / 6}\left(2 / y_{c}\right)^{1 / 3}
$$

where $y_{c}$ is the critical (re-scaled) momentum [46,50,51]. The growth is first driven by the neutrino collisions that suppress the MSW effect. When the asymmetry reaches a value for which the interaction length at the resonance is larger than the resonance width, then the growth starts to be driven by the MSW effect 47,51 that can bring the $\nu_{\alpha}$-asymmetry up to a maximum value of 0.375. A remarkable feature is that the MSW effect is adiabatic for $s^{2} \gtrsim 10^{-9}\left(\mathrm{eV}^{2} /\left|\delta m^{2}\right|\right)^{1 / 4}$ [51]. Below this value the MSW effect becomes non adiabatic and ordinary neutrinos are not converted efficiently into sterile neutrinos anymore. However such a small value of the vacuum mixing angle represents by far the best example of how matter effects can enhance the mixing in vacuum, considering that in the Sun enterior the MSW effect occurs for $s^{2} \gtrsim 10^{-4}$ [52].

One would also like to know which is the upper limit on the vacuum mixing angle for the neutrino asymmetry to be generated. This is a point that has still not been investigated in the literature but we will see, in the next section, that it will prove to be very important for our considerations. Fortunately it is possible to get an analytic estimation. We will be particularly interested to values of $\left|\delta m^{2}\right| \simeq \delta m_{L S N D}^{2} \simeq 1 \mathrm{eV}^{2}$. For these values one can use the Eq. (34) to calculate the sterile neutrino production $N_{\nu_{s}}^{\rho}$. When the sterile neutrino production is negligible $\left(N_{\nu_{s}}^{\rho} \lesssim 0.1\right)$ the value of the critical momentum is approximately given by the peak of Fermi-Dirac distribution: $y_{c} \simeq 2$ [43,44]. Once that the asymmetry generation has started the sterile neutrino production is suppressed in the collision dominated regime. This has the effect that the sterile neutrino production calculated by the Eq. (30) is halved. Thus, taking into account this effect, one can easily calculate that the condition $\Delta N_{\nu_{s}}^{\rho}<0.1$

\footnotetext{
${ }^{10}$ In [48] the results are presented for $\Delta N_{\nu}^{\text {tot }}$, however for small enough mixing angles the contribution to $\Delta N_{\nu}^{f_{\nu}}$ is negligible and a comparison is possible at least for the non resonant case. In the resonant case a comparison with the results of [48], at $s^{2} \geq 10^{-4}$, is not possible because these take into account also the negative contribution $\sum_{\beta}^{e, \mu, \tau} N_{\nu_{\beta}}-3$ to $\Delta N_{\nu}^{\rho}$ and are performed in a quantum kinetic formalism that accounts also for the MSW effect.
} 
corresponds to mixing angles $s^{2}<0.52(0.98) \times 10^{-5} \sqrt{\mathrm{eV}^{2} /\left|\delta m^{2}\right|}$. When this condition is verified, together with the lower limit from the adiabaticity, the final value of the neutrino asymmetry is very close to the impassable limit corresponding to a situation in which, for an initial positive (negative) value of $L^{(\alpha)}$, all anti-neutrinos (neutrinos) are converted into anti-sterile (sterile) neutrinos and thus $\left|L_{\nu_{\alpha}}\right|^{\max }=n_{\nu_{\alpha}}\left(n_{\bar{\nu}_{\alpha}}\right) / n_{\gamma}=3 / 8$ [50]. Therefore the maximum value is also independent on the mixing angle in this range of values. When the sterile neutrino production becomes not negligible $\left(\Delta N_{\nu}^{\rho} \gtrsim 0.1\right)$, it has the effect to delay the asymmetry generation since the value of $y_{c}$ increases and therefore $T_{c}$ decreases. When $y_{c}$ becomes higher than $\sim 10$ the asymmetry generation at the critical temperature is driven by resonant neutrinos well in the tail of the distribution. Thus it is reasonable to think that when this happens the asymmetry generation mechanism will start to turn off. Unfortunately it is not easy to give an analytic description of this effect. However there is a much simpler reason for which the final value of the neutrino asymmetry has to decrease when the sterile neutrino production becomes not negligible. The reason is that the final value is reached during the MSW dominated regime that starts when the asymmetry has become large enough, during the collision dominated regime, that the neutrino and anti-neutrino resonances get completely separated and only anti-neutrino resonance can give a relevant effect, while the neutrino resonance is by far in the tail of the distribution if $L^{(\alpha)}$ is initially positive, vice-versa if it is negative. In this way the MSW effect enhances the asymmetry to its maximum value [50. However if sterile neutrinos have been produced during the previous collision dominated regime, not only ordinary anti-neutrinos will be converted into sterile anti-neutrinos, but also the already produced sterile anti-neutrinos will be converted back into ordinary anti-neutrinos. Thus the maximum value of the final neutrino asymmetry becomes 巴:

$$
\left|L_{\nu_{\alpha}}\right|^{\max }=\frac{3}{8}\left(1-N_{\nu_{s}}^{\rho}\right)
$$

It will prove useful to assume, as upper limit on the mixing angle for the generation of neutrino asymmetry, the value for which $N_{\nu_{s}}^{\rho}>0.9$, corresponding to a final neutrino asymmetry at least one order of magnitude less than its maximum value $3 / 8$. It is easy to calculate this value:

$$
s^{2} \simeq 0.5(1) \times 10^{-4} \sqrt{\mathrm{eV}^{2} /\left|\delta m^{2}\right|}
$$

Let us discuss now the effects of a large neutrino asymmetry. The neutrino asymmetry generation yields two corrections to the $N_{\nu_{s}}^{\rho}$ calculated, in the resonant case, from the Eq. (30). A first correction is due to the effect, just described, of suppression of the sterile neutrino production after the generation of the asymmetry and can be described by a corrective factor to the Eq. (30) that can be as low as 0.5 , for $N_{\nu_{s}}^{\rho} \lesssim 0.1$ and that becomes quickly 1 (no suppression) for $N_{\nu_{s}}^{\rho} \gtrsim 0.1$ [44]. A second effect takes into account the sterile

\footnotetext{
${ }^{11}$ In [44] it has been shown that the distribution function of sterile neutrinos produced during the collision dominated regime, for $y_{c} \gg 1$, is given just by the equilibrium distribution times a coefficient $\alpha \leq 1$ in a way that $n_{\nu_{s}} / n_{\nu}^{\mathrm{eq}}=\rho_{\nu_{s}} / \rho_{\nu}^{\mathrm{eq}}=N_{\nu_{s}}^{\rho}=\alpha$.
} 
neutrino production in the MSW dominated regime that results as an additive contribution to $N_{\nu_{s}}^{\rho}$ from the Eq. (30) that accounts only for the sterile neutrino production in the collision dominated regime. In the calculation of $\Delta N_{\nu}^{\rho}$ one has to take into account also the depletion of ordinary neutrinos. For $-\delta m^{2} \ll 100 \mathrm{eV}^{2}$ the contribution to the sterile neutrino production from the MSW dominated regime occurs below the neutrino chemical decoupling and is compensated by an opposite ordinary neutrino depletion and thus there is no contribution to $\Delta N_{\nu}^{\rho}$. For higher values of $-\delta m^{2}$ ordinary neutrino are re-populated by the annihilations and this second contribution to $\Delta N_{\nu}^{\rho}$ can be as high as 0.4 [53]. However values of $\left|\delta m^{2}\right| \gtrsim 20 \mathrm{eV}^{2}$ are disfavoured by structure formation $+\mathrm{CMB}$ considerations (see for example [15]). In any case the sum of the two contributions to the total $\Delta N_{\nu}^{\rho}$, from the two different regimes, cannot be much higher than 1 . Thus the account of the neutrino asymmetry generation leads only to corrections to the calculation of $\Delta N_{\nu}^{\rho}$.

In the case $\alpha=\mu, \tau$ the contribution $\Delta N_{\nu}^{\rho}$ to $\Delta N_{\nu}^{\text {tot }}$ is the leading effect $\square$ and we can approximately say that the accessible region in the plot $\Delta N_{\nu}^{\text {tot }}-\Delta N_{\nu}^{\rho}$ lies along $\Delta N_{\nu}^{\text {tot }}=$ $\Delta N_{\nu}^{\rho}$ for $0 \leq \Delta N_{\nu}^{\rho} \lesssim 1$ (see figure 2).

In the case $\alpha=e$, a large $\Delta N_{\nu}^{f_{\nu}}$ can arise from two different processes. A first process is the $\nu_{e}, \bar{\nu}_{e}$ number density depletion that this time is a direct and relevant effect occurring for $\left|\delta m^{2}\right| \lesssim 10^{-4} \mathrm{eV}^{2}$ and yields always a positive $\Delta N_{\nu}^{f_{\nu e}}$ that can be even higher than 1 苛. The cosmological constraints are thus strongly strenghtened by the account of this effect [56, 48] and this can be seen in the plot $\Delta N_{\nu}^{\text {tot }}-\Delta N_{\nu}^{\rho}$ considering that the region $\Delta N_{\nu}^{\text {tot }}>\Delta N_{\nu}^{\rho}$ lies largely outside the cosmological allowed region (see figure 2). The second process is the generation of a large electron neutrino asymmetry in the resonant case and at small mixing angles. This time the sign is the same one of the initial $L^{(e)}$ that is observationally unknown and that could be predicted only within a full baryo-leptogenesys model and thus it can be both positive and negative.

For negative values it is remarkable that the region $\Delta N_{\nu}^{\text {tot }}<\Delta N_{\nu}^{\rho}$ becomes accessible in the $\Delta N_{\nu}^{\text {tot }}-\Delta N_{\nu}^{\rho}$ plot. In [53] it has been calculated that $\Delta N_{\nu}^{f_{\nu}}$ can be as low as -1.4 (for $-\delta m^{2} \lesssim 3 \mathrm{eV}^{2}$ ) and in figure 2 one can see that for values $\Delta N_{\nu}^{f_{\nu_{e}}} \lesssim-0.3$, there is compatibility with the region allowed by the low $Y_{p}^{\exp }$ values. Thus $\nu_{e} \leftrightarrow \nu_{s}$ oscillations provide a viable mechanism to solve the claimed SBBN crisis [57,50].

Another interesting possibility, shown in figure 2, is that $\nu_{e} \leftrightarrow \nu_{s}$ oscillations would be also able to reconcile possible future ( $1 \%$ error) values of $\eta^{C M B} \gtrsim 7 \gtrsim \eta^{S B B N}$, with the

\footnotetext{
${ }^{12}$ There is a small positive contribution to $\Delta N_{\nu}^{f_{\nu}}$ at large mixing angles and $\left|\delta m^{2}\right| \lesssim 10^{-4}$ due to a small depletion of $\nu_{e}$ number density induced by the much higher $\nu_{\alpha}$ number density depletion 48, 53.

${ }^{13}$ For example in [54] it is shown that, for $s^{2}=1$ and $\delta m^{2} \simeq 3 \times 10^{-8} \mathrm{eV}^{2}$, the $Y_{p}$ production is 0.02 higher than in SBBN, corresponding to $\Delta N_{\nu}^{\text {tot }} \simeq+1.5$ and implying $\Delta N_{\nu}^{f_{\nu e}}$ at least as high as +0.5 (the value of $\Delta N_{\nu}^{\rho}$ is not separately shown). Extrapolating to higher values of $\left|\delta m^{2}\right|$ it seems also quite evident that much higher values of $\Delta N_{\nu}^{\text {tot }}(3,4, \ldots, ?)$ and of $\Delta N_{\nu}^{f_{\nu_{e}}}(2,3, \ldots, ?)$ are possible. This is confirmed by the results of a very recent work [55] in which it is found that $\left(\Delta N_{\nu}^{\text {tot }}\right)^{\max } \simeq 6$, implying $\Delta N_{\nu}^{f_{\nu e}}$ at least as high as $\simeq 5$.
} 
nuclear abundances observations 4 .

Still another interesting effect could be the possibility to generate the electron neutrino asymmetry in a inhomogeneous way. This would require the presence of small baryon number inhomogeneities [60]. This effect could produce inhomogeneous nuclear abundances that could have two kind of indications as discussed in the previous section: indirectly if one finds $\eta^{C M B}>\eta_{D / H}^{S B B N}$ and $\left(\Delta N_{\nu}^{\rho}\right)^{C M B}<\left(\Delta N_{\nu}\right)^{B B N}$ or, directly, if one finds a dispersion in the values of $(D / H)$ measured from quasar absorption systems $\square$.

Thus the generation of an electron neutrino asymmetry yields many interesting cosmological effects but, within a two neutrino mixing scenario, it appears as a special possibility, considering that it requires $\alpha=e$, negative $\delta m^{2}$ and small mixing angles. However we saw that the generation takes place even for tiny values of the vacuum mixing angles and because of this, the early Universe is the most sensitive way to probe small mixings with new sterile neutrino flavors. Moreover when considering realistic multiflavour mixing scenarios the conditions for the occurrence of an electron neutrino asymmetry generation can be more naturally satisfied.

\section{FOUR NEUTRINO MIXING}

Four neutrino mixing models represent the minimal way to explain, in terms of neutrino oscillations, all three anomalies including the results of the LSND experiment. These models are described by a $4 \times 4$ unitary mixing matrix $U$ connecting the 4 mass eigenstates $\left|\nu_{i}\right\rangle$, with definite masses $m_{i}$, to the 4 flavor eigenstates $\left|\nu_{\alpha}\right\rangle(\alpha=e, \mu, \tau, s)$ :

$$
\left|\nu_{\alpha}\right\rangle=\sum_{i=1}^{4} U_{\alpha i}^{\star}\left|\nu_{i}\right\rangle
$$

There are different possible patterns for the mass spectra but all of them can be distinguished in two types [62 64]. In a first type, the ' $3+1$ ' models, the mass eigenvalue $m_{4}$ is separated by the other three, $m_{i}$, by the LSND gap in a way that $\left|\delta m_{4 i}^{2}\right| \simeq \delta m_{L S N D}^{2}$. This case is a minimal modification of a three neutrino mixing model, since the introduction of a fourth mass eigenstate, to incorporate the LSND results, just perturbs the mixing among the other three explaining solar and atmospheric neutrino results. This means that the fourth eigenstate is almost coinciding with the sterile neutrino flavor $\left(\left|U_{s 4}\right|^{2} \simeq 1,\left|U_{\alpha s}\right|^{2} \ll 1, \alpha \neq s\right)$. In a second type of models, the ' $2+2$ ' models, the spectrum splits in two nearly degenerate pairs with $\left|\delta m^{2}\right|=\delta m_{\odot}^{2}, \delta m_{\mathrm{atm}}^{2}$, separated by the much larger LSND scale $\delta m_{L S N D}^{2}$. In

\footnotetext{
${ }^{14}$ This possibility has been proposed in [58], when the first data from BOOMERanG-MAXIMA were indicating $\eta^{C M B}=9.0 \pm 1.4$ [59].

${ }^{15}$ It is also interesting that inhomogeneous neutrino asymmetries, though on much smaller scales than those necessary to produce inhomogenous nuclear abundances, could be responsible for the generation of galactic magnetic fields and give rise to a detectable cosmological background of gravitational waves 61.
} 
this case the neutrino mixing matrix is very different from the case of three neutrino mixing models.

There is an ongoing debate on which of the two types can better describe the experimental data 63,65 68]. The new data from atmospheric neutrino experiments plus the inclusion of Tritium $\beta$ decay data corner ' $3+1$ ' models in two only allowed regions, at 99\%c.l., around $\delta m_{L S N D}^{2} \sim 0.9$ and $2 \mathrm{eV}^{2}$ 67. On the other hand the fact that both atmospheric 69 $\square$ and solar neutrino [3] data disfavour pure active-sterile oscillations, suggests, for ' $2+2$ ' models, that the $\nu_{e}$ 's (for solar) and the $\nu_{\mu}$ 's (for atmospheric) are converted into some admixture of both active and sterile neutrinos [71,68 . Thus from solar and atmospheric neutrino experiments there is no evidence of the existence of sterile neutrinos and the simplest four neutrino mixing models, predicting pure active to sterile neutrino oscillations, are disfavoured. However there is not uncompatibility among the three experiments when the full range of possible four neutrino mixing models is considered. We will now study the cosmological effects of both ' $3+1$ ' and ' $2+2$ ' class of models.

\section{A. $3+1$ models}

The ' $3+1$ ' models can be distinguished in two classes, $\mathrm{A}$ and $\mathrm{B}$, such that $m_{4} \gg m_{i \neq 4}$ in $\mathrm{A}$ and $m_{4} \ll m_{i \neq 4}$ in $\mathrm{B}$. In the $\mathrm{B}$ case the three heavier mass eigenstates are almost degenerate with $m_{i} \simeq \sqrt{\delta m_{L S N D}^{2}} \simeq 0.95$ or $1.4 \mathrm{eV}$ according to which of the two allowed islands is considered. The Heidelberg-Moscow experiment on $(\beta \beta)_{0 \nu}$ decay puts restrictions on the B class [72]. We can make use of the results seen for $\nu_{\alpha} \leftrightarrow \nu_{s}$ to get some simple estimations on the cosmological output of the two different classes.

Class $A$. One has to consider the different possible ways of oscillations into the sterile neutrino flavor. The sterile neutrino flavor almost coincides with the fourth eigenstate but it is also slightly present in the other three eigenstates. The mixing between the three light eigenstates and the fourth eigenstate is set by $\delta m_{4 i}^{2}$ and, since it is positive, there is no neutrino asymmetry generation. The mixing of the three active neutrinos with the sterile neutrino can be described by three different mixing angles, $\sin ^{2} 2 \theta_{\alpha s} \simeq 4 U_{\alpha 4}^{2}$. For $\alpha=e, \mu$ there are limits from the CDHS and BUGEY experiment for which $\sin ^{2} 2 \theta_{\alpha s} \lesssim 10^{-1}$. For $\alpha=\tau$ we can assume the same limit. Thus from the mixing setted by $\delta m_{L S N D}^{2}$ and using the Eq.'s (30) and (33) one can can see that there is a total thermalization $\left(\Delta N_{\nu}^{\rho}=1\right)$. The LSND experiment relates the two mixing angles in a way that $\sin ^{2} 2 \theta_{e s} \times \sin ^{2} 2 \theta_{\mu s} \simeq 3 \times$ $10^{-4}$. Therefore, even in the case of minimum sterile neutrino production, when $\sin ^{2} 2 \theta_{\text {es }}=$ $\sin ^{2} 2 \theta_{\mu s} \simeq 10^{-2}$ one has $\Delta N_{\nu}^{\rho} \simeq 0.9$, very close to a complete thermalization. A mixing of the three active neutrinos with the sterile neutrino can be also driven by $\delta m_{\odot}^{2}$ and $\delta m_{\text {atm }}^{2}$, since the sterile neutrino is also slightly present in the three light eigenstates. Now the sign of $\delta m^{2}$ can be also negative and thus a neutrino asymmetry generation could occur in principle but the presence of a large sterile neutrino population, from the mixing set by $\delta m_{L S N D}^{2}$, will largely decrease the final value of the asymmetry, at least of one order of magnitude (see the

\footnotetext{
${ }^{16}$ See also [70] for a critical discussion.
} 
Eq. (36) ). In any case such a generation of neutrino asymmetry occurs for $\left|\delta m^{2}\right| \ll 10^{-2} \mathrm{eV}^{2}$ and in this case the critical temperature would be lower than the freezing temperature of the neutron to proton ratio and would not affect BBN predictions in a way that $\left|\Delta N_{\nu}^{f_{\nu e}}\right|$ is negligible. Thus the only relevant cosmological effect is $\Delta N_{\nu}^{\rho} \simeq 0.9$

Class $B$. In this case the mixing of the three quasi-degenerate heavier eigenstates with the fourth eigenstate has a negative $\delta m_{4 i}^{2} \simeq-\delta m_{L S N D}^{2}$. Therefore the sterile neutrino production is of resonant type and from the Eq. (34) with $\left|\delta m^{2}\right| \simeq 1 \mathrm{eV}^{2}$ and $\sin ^{2} 2 \theta \simeq 10^{-2}-10^{-1}$ one can see that again a complete thermalization would occur with $\Delta N_{\nu}^{\rho}$ very close to 1. In principle an electron asymmetry generation can also occur but the complete sterile neutrino thermalization has the effect to suppress completely the asymmetry generation mechanism and thus we can conclude that also in the B case $\left|\Delta N_{\nu}^{f_{\nu_{e}}}\right| \ll 1$ and therefore $\Delta N_{\nu}^{\text {tot }}=\Delta N_{\nu}^{\rho} \simeq 1$.

\section{B. $2+2$ models}

These can be also distinguished in two classes, A and B. In the A (B) class the two lightest mass eigenstates, with masses $m_{1}$ and $m_{2}$, explain solar (atmospheric) neutrino data while the two heavier, with masses $m_{3}$ and $m_{4}$, explain the atmospheric (solar) neutrino data 63, 64]. Let us define simple limit cases in which the lightest and heaviest pair of mass eigenstates are made only of two flavor eigenstates, that means to consider a mixing matrix with two unmixed $2 \times 2$ blocks. Since the atmospheric neutrino experiments constraint the probability of $\nu_{\mu} \rightarrow \nu_{e}$ conversions to be very small, then one has only four different possibilities:

1. The $m_{3}, m_{4}$ mass eigenstates are made only of $\nu_{\mu}, \nu_{\tau}$ and the $m_{1}, m_{2}$ mass eigenstates only of $\nu_{e}, \nu_{s}$.

2. The $m_{3}, m_{4}$ mass eigenstates are made only of $\nu_{e}, \nu_{s}$ and the $m_{1}, m_{2}$ mass eigenstates only of $\nu_{\mu}, \nu_{\tau}$.

3. The $m_{3}, m_{4}$ mass eigenstates are made only of $\nu_{\mu}, \nu_{s}$ and the $m_{1}, m_{2}$ mass eigenstates only of $\nu_{e}, \nu_{\tau}$.

4. The $m_{3}, m_{4}$ mass eigenstates are made only of $\nu_{e}, \nu_{\tau}$ and the $m_{1}, m_{2}$ mass eigenstates only of $\nu_{\mu}, \nu_{s}$.

Note that the 1 and 3 cases belong to the A class, while the 2 and 4 cases belong to the B class. A given neutrino flavor is only contained in one of the two pairs of mass eigenstates, that we call the normal couple, while it is absent in the other one, that we call the opposite couple. It is is simple to calculate the cosmological output since no neutrino asymmetry generation is possible (thus $\Delta N_{\nu}^{f_{\nu_{e}}}=0$ ) and $\Delta N_{\nu}^{\rho}=1$ for the cases 3 and 4 and also for the cases 1 and 2 if the LMA solution is considered for the solar neutrinos ${ }^{7}$, otherwise

\footnotetext{
${ }^{17}$ A LMA solution for a mixing $\nu_{e} \leftrightarrow \nu_{s}$ is excluded by the Homestake experiment but it becomes possible is some mixture of $\nu_{\tau}$ is added to $\nu_{s}$ [68] or if Homestake is disregarded [66].
} 
$\Delta N_{\nu}^{\rho} \simeq 4 \times 10^{-4}$ for the SMA solution $\left(\sin ^{2} 2 \theta_{\mathrm{SMA}} \simeq 10^{-3},\left|\delta m^{2}\right|_{\mathrm{SMA}} \simeq 5 \times 10^{-6} \mathrm{eV}^{2}[38)\right.$ 四.

These simple four limit cases cannot explain the experiments for two reasons. The first reason is that in order to explain the LSND experiment the probability of $\nu_{\mu} \rightarrow \nu_{e}$ conversions cannot vanish and the second reason is that the SK experiment [69] and the SNO experiment [3] disfavour pure active to sterile oscillations. In order to explain the LSND experiment it is necessary that a small mixing between the heavy and light pairs of mass eigenstates is introduced in a way that there is a small contamination of $\nu_{e}$ and $\nu_{\mu}$ also in the respective opposite couple 63,64. In order to explain the SK and SNO results one has to introduce a parameter that allows also for $\nu_{e} \rightarrow \nu_{\mu, \tau}$ conversions 19 in the case 1 and 2 and for $\nu_{\mu} \rightarrow \nu_{\tau}$ in the cases 2 and 4 . This is usually done introducing a mixing angle between the sterile and $\nu_{\mu, \tau}$ in a way that $\left(\nu_{\mu, \tau}, \nu_{s}\right) \rightarrow\left(\nu_{\mu, \tau}^{\prime}, \nu_{s}^{\prime}\right)=U(\alpha)\left(\nu_{\mu, \tau}, \nu_{s}\right)$, where $U(\alpha)$ is a $2 \times 2$ rotation matrix 64. In this way the 1 and 2 cases correspond to $\alpha=0$, while the 3 and 4 cases correspond to $\alpha=\pi / 2$ and for $\alpha=0 \rightarrow \pi / 2$ there is a continuous transformation bringing from 1 to 3 and from 2 to 4 .

Class $A$. Let us first consider the transformation from 1 to 3 . It is remarkable that when the condition for adiabaticity is satisfied for very small mixing angles $\sin ^{2} \alpha \gtrsim$ $10^{-9}\left(\mathrm{eV}^{2} /\left|\delta m^{2}\right|\right)^{1 / 4}$, a large muon-tauon neutrino asymmetry can be generated. This asymmetry generation can both suppress the sterile neutrino production from $\nu_{e} \rightarrow \nu_{s}$ but also be partly transferred into an electron neutrino asymmetry yielding $\Delta N_{\nu}^{f_{\nu_{e}}} \simeq-0.3$ or $\Delta N_{\nu}^{f_{\nu e}} \simeq 0.1$, according on the sign of the initial total asymmetry $L^{(\mu, \tau)}$ [73]. However for $\sin ^{2} 2 \alpha \gtrsim 10^{-4}$ (see Eq. (37)), the mixing $\nu_{\mu, \tau} \leftrightarrow \nu_{s}$ with $\delta m^{2} \simeq-\delta m_{L S N D}^{2}$, produces a sterile neutrino production $\Delta N_{\nu}^{\rho} \gtrsim 0.9$ that suppresses a large neutrino asymmetry generation and the final $\left|\Delta N_{\nu}^{f_{\nu_{e}}}\right| \ll 0.1$. The experimental data favour, like best fits, $\sin ^{2} \alpha \simeq 0.03-0.09$ and $\sin ^{2} \alpha=0.80-0.82$ 68 20 and disfavour the possibility to have $\alpha$ (and also $\alpha^{\prime} \equiv \pi / 2-\alpha$ ) smaller than $10^{-4}$. Therefore the cosmological effects are a resonant sterile neutrino production with $\Delta N_{\nu}^{\rho} \simeq 1$ and $\left|\Delta N_{\nu}^{f_{\nu e}}\right| \ll 1$ for $\sin ^{2} \alpha$ around the range $0.03-0.09$ and a non resonant sterile neutrino production with $\Delta N_{\nu}^{\rho} \simeq 1$ and $\left|\Delta N_{\nu}^{f_{\nu_{e}}}\right|=0$ for $\sin ^{2} \alpha$ around $0.80-0.82$. Note that the result is always $\Delta N_{\nu}^{\rho} \simeq 1$, even assuming a SMA solution to solve the solar neutrino problem. In figure 3 the approximate accessible region for the class $\mathrm{A}$ ' $2+2$ ' models and for $\sin ^{2} \alpha \gtrsim 10^{-9}\left(\mathrm{eV}^{2} /\left|\delta m^{2}\right|\right)^{1 / 4}$ is shown in the $\Delta N_{\nu}^{\text {tot }}-\Delta N_{\nu}^{\rho}$ plot with thick solid lines. The experimental results from the SNO and SK experiments corner it to the 'point' $\Delta N_{\nu}^{\rho}=\Delta N_{\nu}^{\text {tot }} \simeq 1$, represented as a small circle.

Class $B$. The other possibility is to consider a transition from the case 4 to the case 2 for $\alpha^{\prime}=\pi / 2-\alpha=0 \rightarrow \pi / 2$. Again when $\sin ^{2} 2 \alpha^{\prime}$ becomes $\gtrsim 10^{-9}\left(\mathrm{eV}^{2} /\left|\delta m^{2}\right|\right)^{1 / 4}$ a neutrino asymmetry generation occurs and this inhibits the sterile neutrino production from $\nu_{\mu} \leftrightarrow \nu_{s}$

\footnotetext{
${ }^{18}$ The SMA solution cannot give an electron neutrino asymmetry generation because $\delta m^{2}$ is positive.

${ }^{19}$ With $\nu_{\mu, \tau}$ we indicate a mixture of $\nu_{\mu}$ and $\nu_{\tau}$. This further mixing has no relevance in cosmology, since the $\nu_{\mu}$ and $\nu_{\tau}$ flavors cannot be distinguished.

${ }^{20}$ In the notation of $68 \cos \alpha=c_{23} c_{24}$.
} 
27. However again the SNO and SK experiments favour $\sin ^{2} 2 \alpha^{\prime} \gg 10^{-4}$ and the consequent large sterile neutrino production prevents a large neutrino asymmetry to be generated and again $\Delta N_{\nu}^{\rho} \simeq \Delta N_{\nu}^{\text {tot }} \simeq 1$. In figure 3 we again show a plausible accessible region in the $\Delta N_{\nu}^{\text {tot }}-\Delta N_{\nu}^{\rho}$ plot. Since in the case of asymmetry generation there are no calculations of $\left|\Delta N_{\nu}^{f_{\nu}}\right|$, we show the most conservative region (between the dashed lines) assuming that $\left|\Delta N_{\nu}^{f_{\nu}}\right|$ can take all values between zero and the maximum possible value. This value corresponds to the case of an electron neutrino asymmetry generation in the limit of two neutrino mixing $\nu_{e} \leftrightarrow \nu_{s}$, for $-\delta m^{2} \simeq 1 \mathrm{eV}^{2}$, as calculated in [53].

Let us try to quantify to which confidence level the results found in [68] constraint the possibility to have very small mixing angle $\sin ^{2} \alpha \lesssim 10^{-4}$ or $\sin ^{2} 2 \alpha^{\prime} \lesssim 10^{-4}$, that is equivalent to exclude the possibility of a neutrino asymmetry generation respectively in the A class and in the B class. This depends on which solution one assumes for the solar neutrino data. If one assumes a LMA solution then the best fit is for $\sin ^{2} \alpha=0.80-0.82$ or equivalently for $\sin ^{2} \alpha^{\prime}=0.18-0.2$. Very small values $\sin ^{2} 2 \alpha^{\prime} \lesssim 10^{-4}$ are excluded approximately at $95 \%$ c.l. If one assumes a SMA solution then the best fit is for $\sin ^{2} \alpha=$ $0.03-0.09$. In this case very small values $\sin ^{2} \alpha \lesssim 10^{-4}$ are very slightly disfavoured and cannot be excluded to a significant statistical confidence level. However, from the reported values of $\chi_{\min }^{2}$, the first case, assuming the LMA solution, is favoured compared to the second case, assuming the SMA solution, and thus values of $\sin ^{2} \alpha \lesssim 10^{-4}$ are disfavoured approximately at the $90 \%$ c.l.. In the next year the SNO experiment should be able to constraint more significantly pure active to sterile neutrino oscillations in solar neutrinos and in particular the case when the SMA solution is considered, unless evidence for $\nu_{e} \rightarrow \nu_{s}$ will be found.

Therefore we arrive to the conclusion that current experiments favour those four neutrino mixing models, both of $3+1$ and 2+2 type, in which the sterile neutrino flavor is brought to a complete, or almost complete, thermalization and no large electron asymmetry generation is possible in a way that the final result is always $\Delta N_{\nu}^{\rho}=\Delta N_{\nu}^{\text {tot }} \simeq 1$. Therefore, from the upper limit $\Delta N_{\nu}^{\text {tot }} \leq 0.3$, current cosmological observations disfavour 4 neutrino mixing models. There are however some possibilities for which the cosmological bound could be evaded.

- systematic uncertainties or statistical errors have been underestimated in $Y_{p}$ and/or $\eta^{C M B}$ measurements. In the case of higher $Y_{p}$ and/or lower $\eta^{C M B}$ then $\Delta N_{\nu}^{\text {tot }}=1$ could be possible. For example one total extra neutrino species would be allowed at $3 \sigma$ in the case of underestimated systematic uncertainties if: $Y_{p}=0.254 \pm 0.002$ and $\eta^{C M B}$ unchanged as in the (2) or if $\eta^{C M B}=3.5_{-0.8}^{+1.1}$ and high values $Y_{p}^{\exp }$ are used; in the case of underestimated statistical errors if $Y_{p}^{\exp }=0.244 \pm 0.006$ and $\eta^{C M B}$ as in the (2) or if $\eta^{C M B}=6.0 \pm 1.5$ and high values of $Y_{p}^{\exp }$ are used ${ }^{2}$.

${ }^{21}$ Calculations of $\left|\Delta N_{\nu}^{f_{\nu_{e}}}\right|$ are missing in this case.

${ }^{22}$ These are qualitative estimations because we are calculating the $99 \%$ c.l. just multiplying by a factor 3 the error at $68 \%$ c.l., as for a gaussian distribution. This is a very rough assumption when $\delta \eta / \eta$ is not $\ll 1$ and a more elaborate statistical procedure should be used. 
- We assumed small initial neutrino asymmetries. If some unknown mechanism created large neutrino asymmetries $\left(\sim 10^{-5}-10^{-4}\right)$ above $T \sim 15 \mathrm{MeV}$ (the characteristic temperature for oscillations with $\left|\delta m^{2}\right|=\delta m_{L S N D}^{2} \sim 1 \mathrm{eV}^{2}$ ), then the sterile neutrino production would be completely suppressed [43]. In this case the constraints that we obtained in section 2 should be applied to the values of $\Delta N_{\nu}^{\rho}$ and $\Delta N_{\nu}^{f_{\nu e}}$ associated to large neutrino asymmetries (see Eq. (26) ).

- We neglected completely the presence of phases in the four neutrino mixing matrix since we used the analogy with two neutrino mixing to calculate the cosmological output. The role of phases in cosmology has never been studied. A possibility could be that, when phases are taken into account, then the active-sterile neutrino mixing, even with large angles $\sin ^{2} 2 \alpha \gtrsim 10^{-4}$, can generate a large neutrino asymmetry that suppresses the sterile neutrino production, or in the case of an electron neutrino asymmetry, yields a negative $\Delta N_{\nu}^{f_{\nu}}$. This possibility should be verified in a full 4 neutrino mixing kinetic theory.

\section{FIVE NEUTRINO MIXING}

If one assumes the existence of a mixing with a second light sterile neutrino flavor $\nu_{s^{\prime}}$ then it is possible to evade the cosmological bound if the mixing generates a large neutrino asymmetry able to suppress the production of the first sterile neutrino and in the case of an electron neutrino asymmetry also to yield a large negative $\Delta N_{\nu}^{f_{\nu e}}$. This new mass eigenstate should be added to the four neutrino mixing solutions that explain the experiments and that we described in the previous section. For convenience let us refer to the first neutrino flavor as the LSND neutrino. It is necessary to distinguish between models in which the LSND neutrino production is resonant and models in which it is not resonant.

In the non resonant case, even though the asymmetry can start to be generated by the mixing with the $s^{\prime}$-neutrino, afterwards it gets destroyed by the mixing with the LSND neutrino [43,44]. Thus the addition of a second sterile neutrino flavour to the A class ' $3+1$ ' type models and to the ' $2+2$ ' models that are close to the limit cases 2 and 3 cannot help to evade the cosmological bound.

In the resonant case the generation of a neutrino asymmetry from the mixing with $\nu_{s^{\prime}}$ is not obstacled by the mixing with the LSND neutrino. Thus any $\alpha$-neutrino asymmetry generation has the effect to suppress the sterile neutrino production. However there cannot be a full suppression, because necessarily $\left|\delta m_{\alpha s^{\prime}}^{2}\right| \simeq\left|\delta m_{\alpha s}^{2}\right|$ and the asymmetry generation starts when already about half of the sterile neutrino production occurred and the final result is $\Delta N_{\nu}^{\rho} \simeq 0.5$. This is the only effect in the case of generation of a muon or tauon neutrino asymmetry and thus $\Delta N_{\nu}^{\text {tot }}=\Delta N_{\nu}^{\rho} \simeq 0.5$. This means that adding a second sterile neutrino flavor to the ' $2+2$ ' models 'close' to the limit case 1 (those for $\sin ^{2} \alpha \simeq 0.05$ ) improves the agreement with cosmology but still not within $3 \sigma$. In the case of an electron asymmetry generation one can have also a negative contribution from $\Delta N_{\nu}^{f_{\nu_{e}}}$ and the cosmological bound can be fully evaded. This means that the addition of a second sterile neutrino flavor makes possible to evade the cosmological bound only when it is added to four neutrino spectra of type ' $3+1$ ' class $B$ or ' $2+2$ ' models close to the limit case $4\left(\sin ^{2} \alpha \simeq 0.80\right)$, 
in which the LSND neutrino is present mainly in the light pair of mass eigenstates and an electron neutrino asymmetry can be generated. This possibility to evade the bound can be tested both with future $\beta \beta_{0 \nu}$ decay experiments but also with future cosmological $\mathrm{CMB}$ observations that should find $\left(\Delta N_{\nu}^{\rho}\right)^{C M B} \simeq 0.5$. Moreover one should also have $\eta^{C M B}>\eta_{D / H}^{S B B N}$, but considering the current error on $D / H$ measurement, this possibility can be distinguished only if future CMB observations will give $\eta^{C M B} \gtrsim 7.7$ (at $3 \sigma$ ).

\section{DISCUSSION AND CONCLUSIONS}

We described an analytical and graphical procedure to search for non standard effects from nuclear abundances and CMB obervations. The recent measurement of the baryon content from CMB anisotropies improves remarkably the cosmological information on new physics. The present observations do not show evidences of the presence of non standard effects and constraints can be conveniently displayed in the $\Delta N_{\nu}^{\text {tot }}-\Delta N_{\nu}^{\rho}$ plot.

However future measurements of $\eta$ and $\Delta N_{\nu}^{\rho}$ from CMB, together with the current measurements of primordial Helium- 4 and Deuterium nuclear abundances, could provide some interesting signatures. Here is a summary list of the possible signatures as we found in the second section.

1. If $\eta^{C M B} \gtrsim 7$ then $\Delta N_{\nu}^{\text {tot }}<0$ and a negative $\Delta N_{\nu}^{f_{\nu e}}$ can be invoked.

2. If $\eta^{C M B} \gtrsim 7.7$ then also $\left(\Delta N_{\nu}^{\rho}\right)^{B B N}>0$.

3. If $\left(\Delta N_{\nu}^{\rho}\right)^{C M B}>0$ then $\left(\Delta N_{\nu}^{\rho}\right)^{B B N}>0$ if one can exclude massive neutrino decays or other exotic effects intervening between the BBN and recombination epochs.

4. If $\left(\Delta N_{\nu}^{\rho}\right)^{B B N} \gtrsim 0.3$ then, from the bound $\Delta N_{\nu}^{\text {tot }}<0.3$, one can conclude that $\Delta N_{\nu}^{f_{\nu_{e}}}<$ 0 .

5. If the point 2 is verified but $\left(\Delta N_{\nu}^{\rho}\right)^{C M B}<\left(\Delta N_{\nu}^{\rho}\right)^{B B N}$, then this can be interpreted as a signature of inhomogenous $\Delta N_{\nu}^{f_{\nu}}$. This should be confirmed by inhomogeneities in $(D / H)$ measurements that should be anyway searched independently on CMB observations.

We have applied these cosmological tools to the search of non standard effects from neutrino mixing. In the case of three ordinary neutrino mixing it seems not possible to find relevant cosmological effects. When a mixing with new light sterile neutrino flavors is considered, like the LSND experiment seems to suggest, then the early Universe becomes a powerful probe. We have shown how the SNO and SK experiments favour those four neutrino mixing models for which the sterile neutrino flavor is brought into thermal equilibrium or very close to it, implying that $\Delta N_{\nu}^{\rho} \simeq 1$. At the same time a mechanism of electron neutrino asymmetry generation cannot be invoked to have a negative $\Delta N_{\nu}^{f_{\nu}}$ and thus in the end $\Delta N_{\nu}^{\text {tot }} \simeq 1$. The cosmological bound $\Delta N_{\nu}^{\text {tot }}<0.3$ is already quite conservative and future cosmological observations will be compatible with $\Delta N_{\nu}^{\text {tot }} \simeq 1$ only if they will measure a value for $\eta^{C M B}$ that should be approximately half than the value measured by current observations or, alternatively, a value of $Y_{p}$ that should be about 0.01 higher. This of course 
would mean that present observations are affected by large systematic uncertainties or that statistical errors have been largely underestimated. If one excludes such a possibility then a way out could be the presence of large initial neutrino asymmetries suppressing the sterile neutrino production. In this case the cosmological information can still be used to constraint the values of the neutrino asymmetries. Such a conclusion would have a quite remarkable impact on baryo-leptogenesis models. Another possibility is that phases in the neutrino mixing matrix could play an important role in the derivation of cosmological output and thus should be taken into account. Another intriguing possibility is to assume the existence of more than one sterile neutrino flavor. The new sterile neutrino flavor should be mixed with the electron neutrino flavor with the proper mixing parameters such that a relevant electron neutrino asymmetry is generated and both halves the sterile neutrino production and yields a negative $\Delta N_{\nu}^{f_{\nu e}}$. This is possible only if the electron neutrino flavor is mainly present in the heavy mass eigenstates with $m_{i} \sim 1 \mathrm{eV}$. Therefore this scenario will be testable in future $\beta \beta_{0 \nu}$ decay experiments and with the cosmological tools that we described.

This investigation thus shows that light sterile neutrinos in cosmology are now more constrained than before, because the possibility of a neutrino asymmetry generation in four neutrino mixing models is disfavoured within the statistical significance of the results from the SNO [3] and the SK [69] experiments. The result is that the sterile neutrino flavor, required by the LSND experiment, gets fully thermalized. Therefore the confirmation of the existence of light sterile neutrino flavors in next neutrino mixing experiments would be of great relevance for cosmology. Such a confirmation should come in next years by many planned experiments. In particular the MiniBooNE experiment should confirm or disprove the evidence of neutrino oscillations in the LSND experiment, while many other different experiments will be able to exclude exotic solutions to explain solar and atmospheric neutrino data.

\section{Acknowledgements}

The author is an Alexander von Humboldt research fellow. He wishes to thank L. Mersini for many valuable comments and discussions. He also thanks R. Foot and R.R. Volkas for encouraging comments, M.C. Gonzalez-Garcia for a useful discussion during the EPS HEP 2001 conference in Budapest, M. Tegmark and X. Wang for explanations on CMB data analysis, Q. Shafi and A. Ringwald for nice discussions. He is grateful to M. Lusignoli for having drawn to his attention important points on the statistical significance of current four neutrino mixing data analysis in excluding pure active to sterile neutrino oscillations. 


\section{REFERENCES}

[1] Y. Fukuda et al., Super-Kamiokande Coll., Phys. Rev. Lett. 81 (1998) 1562; Phys. Rev. Lett. 82, 2644 (1999); Y. Fukuda et al., Kamiokande Coll., Phys. Lett. B 335 (1994) 237; R. Becker-Szendy et al., IMB Coll., Nucl. Phys. B (Proc. Suppl.) 38 (1995) 331; W.W.M. Allison et al., Soudan Coll., Phys. Lett. B 449 (1999) 137; M. Ambrosio et al., MACRO Coll., Phys. Lett. B 434 (1998) 451.C. McGrew in Neutrino Telescopes 2001, Venice, Italy, March 2001, to appear; T. Toshito in Moriond 2001, Les Arcs, France, March 2001, to appear. For a review see T. Kajita and Y. Totsuka, Rev. Mod. Phys. 73 (2001) 85.

[2] B.T. Cleveland et al., Astrophys. J. 496 (1998) 505; K.S. Hirata et al., Kamiokande Coll., Phys. Rev. Lett. 77 (1996) 1683; W. Hampel et al., GALLEX Coll., Phys. Lett. B 447 (1999) 127; D.N. Abdurashitov et al., SAGE Coll., Phys. Rev. Lett. 83 (1999) 4686; Y. Fukuda et al., Super-Kamiokande Coll., Phys. Rev. Lett. 81 (1998) 1158.

[3] Q. R. Ahmad et al. [SNO Collaboration], nucl-ex/0106015.

[4] P. Lipari and M. Lusignoli, Phys. Rev. D 60 (1999) 013003 hep-ph/9901350. G. L. Fogli, E. Lisi, A. Marrone and G. Scioscia, Phys. Rev. D 60 (1999) 053006 hep-ph/9904248; N. Fornengo, M. Maltoni, R. Tomàs Bayo and J. W. F. Valle, hep$\mathrm{ph} / 0108043$.

[5] A. Aguilar et al. [LSND Collaboration], hep-ex/0104049.

[6] V. F. Shvartsman, Pisma Zh. Eksp. Teor. Fiz. 9 (1969) 315 [JETP Lett. 9 (1969) 184].

[7] G. Steigman, D. N. Schramm and J. R. Gunn, Phys. Lett. B 66 (1977) 202.

[8] P. de Bernardis et al., Nature 404 (2000) 955 [astro-ph/0004404; S. Hanany et al., Astrophys. J. 545 (2000) L5 astro-ph/0005123; N. W. Halverson et al., astro-ph/0104489.

[9] A. E. Lange et al., Phys. Rev. D 63 (2001) 042001 astro-ph/0005004; A. Balbi et al., Astrophys. J. 545 (2000) L1 astro-ph/0005124.

[10] C. B. Netterfield et al., astro-ph/0104460.

[11] C. Pryke et al., astro-ph/0104490.

[12] R. Stompor et al., astro-ph/0105062.

[13] http://map.gsfc.nasa.gov/m_mm/ms_status.html

[14] http://astro.estec.esa.nl/Planck/

[15] X. Wang, M. Tegmark and M. Zaldarriaga, astro-ph/0105091.

[16] M. Tegmark, private communication.

[17] R. V. Wagoner, W. A. Fowler and F. Hoyle, Astrophys. J. 148 (1967) 3; M. S. Smith, L. H. Kawano and R. A. Malaney, Astrophys. J. Supp. 85 (1993) 219.

[18] Y.I. Izotov and T.X. Thuan, Ap.J. 500, 188 (1998).

[19] K. A. Olive and G. Steigman, Astrophys. J. Supp. 97 (1995) 49 astro-ph/9405022.

[20] J. M. O’Meara, D. Tytler, D. Kirkman, N. Suzuki, J. X. Prochaska, D. Lubin and A. M. Wolfe, astro-ph/0011179.

[21] R. E. Lopez and M. S. Turner, Phys. Rev. D 59 (1999) 103502 astro-ph/9807279.

[22] T. P. Walker, G. Steigman, D. N. Schramm, K. A. Olive and H. Kang, Astrophys. J. 376 (1991) 51.

[23] S. Sarkar, Rept. Prog. Phys. 59 (1996) 1493 hep-ph/9602260.

[24] A. D. Dolgov, S. H. Hansen and D. V. Semikoz, Nucl. Phys. B 503 (1997) 426 hepph/9703315]. 
[25] E. Lisi, S. Sarkar and F. L. Villante, Phys. Rev. D 59 (1999) 123520 hep-ph/9901404.

[26] Z. Fodor, S. D. Katz and A. Ringwald, hep-ph/0105064.

[27] H. Kurki-Suonio and E. Sihvola, Phys. Rev. D 63 (2001) 083508 astro-ph/0011544.

[28] G. Jungman, M. Kamionkowski, A. Kosowsky and D. N. Spergel, Phys. Rev. D 54 (1996) 1332 astro-ph/9512139; J. R. Bond, G. Efstathiou and M. Tegmark, Mon.Not.R.Astron.Soc. 291, 33 (1997).

[29] N. Hata, R. J. Scherrer, G. Steigman, D. Thomas, T. P. Walker, S. Bludman and P. Langacker, Phys. Rev. Lett. 75 (1995) 3977 [hep-ph/9505319].

[30] S. Hannestad, astro-ph/0105220.

[31] R. E. Lopez, S. Dodelson, A. Heckler and M. S. Turner, Phys. Rev. Lett. 82 (1999) 3952 astro-ph/9803095.

[32] S. Hannestad, Phys. Rev. Lett. 85 (2000) 4203 astro-ph/0005018].

[33] A. D. Dolgov and B. E. Pagel, New Astron. 4 (1999) 223 astro-ph/9711202].

[34] H. Reeves, Phys. Rev. D 6 (1972) 3363; A. Yahil and G. Beaudet, Astrophys. J. 206 (1976) 26; G. Beaudet and P. Goret, Astron. \& Astrophys. 49, 415 (1976); K. A. Olive, D. N. Schramm, D. Thomas and T. P. Walker, Phys. Lett. B 265 (1991) 239; H. Kang and G. Steigman, Nucl. Phys. B 372 (1992) 494.

[35] J. P. Kneller, R. J. Scherrer, G. Steigman and T. P. Walker, astro-ph/0101386; J. Lesgourgues and A. R. Liddle, astro-ph/0105361. S. H. Hansen, G. Mangano, A. Melchiorri, G. Miele and O. Pisanti, astro-ph/0105385.

[36] M. Apollonio et al. [CHOOZ Collaboration], Phys. Lett. B 466 (1999) 415 hepex/9907037.

[37] G. L. Fogli, E. Lisi, D. Montanino and A. Palazzo, hep-ph/0106247; A. Bandyopadhyay, S. Choubey, S. Goswami and K. Kar, arXiv:hep-ph/0106264.

[38] J. N. Bahcall, M. C. Gonzalez-Garcia and C. Pena-Garay, JHEP 0108 (2001) 014 hhepph/0106258].

[39] F. Vissani, hep-ph/9708483; V. Barger, S. Pakvasa, T. J. Weiler and K. Whisnant, Phys. Lett. B 437 (1998) 107 hep-ph/9806387]. A. J. Baltz, A. S. Goldhaber and M. Goldhaber, Phys. Rev. Lett. 81 (1998) 5730 hep-ph/9806540; G. Altarelli and F. Feruglio, Phys. Lett. B 439 (1998) 112 hhep-ph/9807353].

[40] P. Langacker, S. T. Petcov, G. Steigman and S. Toshev, Nucl. Phys. B 282 (1987) 589.

[41] J. T. Peltoniemi, D. Tommasini and J. W. Valle, Phys. Lett. B 298 (1993) 383; J. T. Peltoniemi and J. W. Valle, Nucl. Phys. B 406 (1993) 409 hep-ph/9302316; D. O. Caldwell and R. N. Mohapatra, Phys. Rev. D 48 (1993) 3259.

[42] D. E. Groom et al. [Particle Data Group Collaboration], Eur. Phys. J. C 15 (2000) 1.

[43] R. Foot and R. R. Volkas, Phys. Rev. Lett. 75 (1995) 4350 [hep-ph/9508275].

[44] P. Di Bari, P. Lipari and M. Lusignoli, Int. J. Mod. Phys. A 15 (2000) 2289 hepph/9907548].

[45] J. M. Cline, Phys. Rev. Lett. 68 (1992) 3137.

[46] R. Foot, M. J. Thomson and R. R. Volkas, Phys. Rev. D 53 (1996) 5349 hepph/9509327]; R. Foot and R. R. Volkas, Phys. Rev. D 55, 5147 (1997) hep-ph/9610229].

[47] P. Di Bari, R. Foot, R. R. Volkas and Y. Y. Wong, hep-ph/0008245.

[48] K. Enqvist, K. Kainulainen and M. Thomson, Nucl. Phys. B 373 (1992) 498.

[49] A. D. Dolgov, Phys. Lett. B 506 (2001) 7 hep-ph/0006103.

[50] R. Foot and R. R. Volkas, Phys. Rev. D 56, 6653 (1997) [hep-ph/9706242]. 
[51] P. Di Bari and R. Foot, hep-ph/0103192.

[52] S. P. Mikheev and A. Y. Smirnov, Nuovo Cim. C 9 (1986) 17; Sov. J. Nucl. Phys. 42 (1985) 913 [Yad. Fiz. 42 (1985) 1441].

[53] R. Foot, Phys. Rev. D 61 (2000) 023516 hep-ph/9906311.

[54] D. P. Kirilova and M. V. Chizhov, Nucl. Phys. B 591 (2000) 457 [hep-ph/9909408].

[55] D. P. Kirilova, astro-ph/0109105.

[56] K. Kainulainen, Phys. Lett. B 244 (1990) 191; R. Barbieri and A. Dolgov, Phys. Lett. B 237 (1990) 440. X. Shi, D. N. Schramm and B. D. Fields, Phys. Rev. D 48 (1993) 2563 astro-ph/9307027]. D. P. Kirilova and M. V. Chizhov, Phys. Lett. B 393 (1997) 375 hep-ph/9608270];

[57] X. Shi, Phys. Rev. D 54 (1996) 2753 arXiv:astro-ph/9602135.

[58] P. Di Bari and R. Foot, Phys. Rev. D 63 (2001) 043008 [hep-ph/0008258].

[59] A.H. Jaffe et.al., Phys. Rev. Lett. 86 (2001) 3475.

[60] P. Di Bari, Phys. Lett. B 482 (2000) 150 [hep-ph/9911214].

[61] A. D. Dolgov and D. Grasso, astro-ph/0106154.

[62] N. Okada and O. Yasuda, Int. J. Mod. Phys. A 12 (1997) 3669 hep-ph/9606411.

[63] S. M. Bilenkii, C. Giunti and W. Grimus, Eur. Phys. J. C 1 (1998) 247 hep-ph/9607372;

[64] V. Barger, S. Pakvasa, T. J. Weiler and K. Whisnant, Phys. Rev. D 58 (1998) 093016 hep-ph/9806328.

[65] S. M. Bilenkii, C. Giunti, W. Grimus and T. Schwetz, Phys. Rev. D 60 (1999) 073007 hep-ph/9903454]; C. Giunti and M. Laveder, JHEP 0102 (2001) 001 hep-ph/0010009]; O. L. Peres and A. Y. Smirnov, Nucl. Phys. B 599 (2001) 3 hep-ph/0011054; W. Grimus and T. Schwetz, Eur. Phys. J. C 20 (2001) 1 [hep-ph/0102252].

[66] V. Barger, B. Kayser, J. Learned, T. Weiler and K. Whisnant, Phys. Lett. B 489 (2000) 345 hhep-ph/0008019];

[67] M. Maltoni, T. Schwetz and J. W. Valle, hep-ph/0107150;

[68] M. C. Gonzalez-Garcia, M. Maltoni and C. Pena-Garay, hep-ph/0108073. For earlier works (pre-SNO results [3]) see for example: C. Giunti, M. C. Gonzalez-Garcia and C. Pena-Garay, Phys. Rev. D 62 (2000) 013005 hep-ph/0001101]; O. Yasuda, hepph/0006319; M. Hirsch and J. W. Valle, Phys. Lett. B 495 (2000) 121 [hep-ph/0009066].

[69] S. Fukuda et al. [Super-Kamiokande Collaboration], Phys. Rev. Lett. 85 (2000) 3999 hep-ex/0009001.

[70] R. Foot, Phys. Lett. B 496 (2000) 169 hep-ph/0007065.

[71] V. Barger, D. Marfatia and K. Whisnant, hep-ph/0106207.

[72] H. V. Klapdor-Kleingrothaus, H. Pas and A. Y. Smirnov, Phys. Rev. D 63 (2001) 073005 hep-ph/0003219; S. M. Bilenky, S. Pascoli and S. T. Petcov, hep-ph/0104218.

[73] N. F. Bell, R. Foot and R. R. Volkas, Phys. Rev. D 58 (1998) 105010 hep-ph/9805259. 


\section{Figure Captions}

Figure 1. Constraints on non standard BBN models from measurements of $\eta^{(C M B)}, Y_{p}$ and $(D / H)$. The solid vertical lines are the constraints (23) and (24) with the thick ones indicating the joint range coming from low + high $Y_{p}$ values. The horizontal solid lines is the constraint (25). The dark gray region is the allowed one by current observations. The dashed lines, contours of the gray region, are the constraints obtained neglecting the error on $\eta^{C M B}$ in the BBN predictions and assuming a value $\eta^{(C M B)}=\eta^{S B B N}=5.6$, corresponding to $(D / H)=3.0 \times 10^{-5}$ in $\mathrm{SBBN}$. The light gray region is the allowed region if one assumes $\eta^{C M B}=7$ and low + high $Y_{p}^{\exp }$ range of values. The dotted horizontal lines are the realistic constraints that will be obtained on $\Delta N_{\nu}^{\rho}$ from future CMB anisotropy observations. The BBN from Standard Model of Particle Physics lies well within the circle centered around the origin.

Figure 2. Accessible region for $\nu_{\alpha} \leftrightarrow \nu_{s}$ in the plane $\Delta N_{\nu}^{\text {tot }}-\Delta N_{\nu}^{\rho}$. The thick segment along the line $\Delta N_{\nu}^{\text {tot }}=\Delta N_{\nu}^{\rho}$ corresponds to the case $\alpha=\mu, \tau$, while the striped regions are for the case $\alpha=e$. The solid striped region is accessible in the case of $\nu_{e}, \bar{\nu}_{e}$ number density depletion below the neutrino chemical decoupling or in the case of negative electron asymmetry generation $\left(\Delta N_{\nu}^{f_{\nu}} \geq 0\right)$ plus sterile neutrino production $\left(\Delta N_{\nu}^{\rho} \geq 0\right)$. The dashed striped region is accessible when a large positive electron neutrino asymmetry is generated. The thick dashed segment for $\Delta N_{\nu}^{\rho}=0$ corresponds to the region of mixing parameters for which the sterile neutrino production in the collision dominated regime is negligible. The possibility to have both an asymmetry generation and a sterile neutrino production $\left(\Delta N_{\nu}^{f_{\nu e}} \neq 0, \Delta N_{\nu}^{\rho}>0\right)$ has not been studied in detail and there are only particular numerical examples. The dotted line is a simple interpolation between the two extreme cases $\Delta N_{\nu}^{\rho}=0$ and $\Delta N_{\nu}^{\rho}=1$ that provides a reasonable approximation.

Figure 3. Approximate accessible regions in the $\Delta N_{\nu}^{\text {tot }}-\Delta N_{\nu}^{\rho}$ plot, in the case of four neutrino mixing models. The solid lines are for A class ' $2+2$ ' models. The region between the dashed lines constraints B class ' $2+2$ ' models. The dotted segment is for the A class ' $3+1$ ' model. The small circle is for the B class ' $3+1$ ' model and for all ' $2+2$ ' models when the information from SNO and SK is used and it can be seen that it lies well outside the allowed cosmological region (in gray). 
figure1

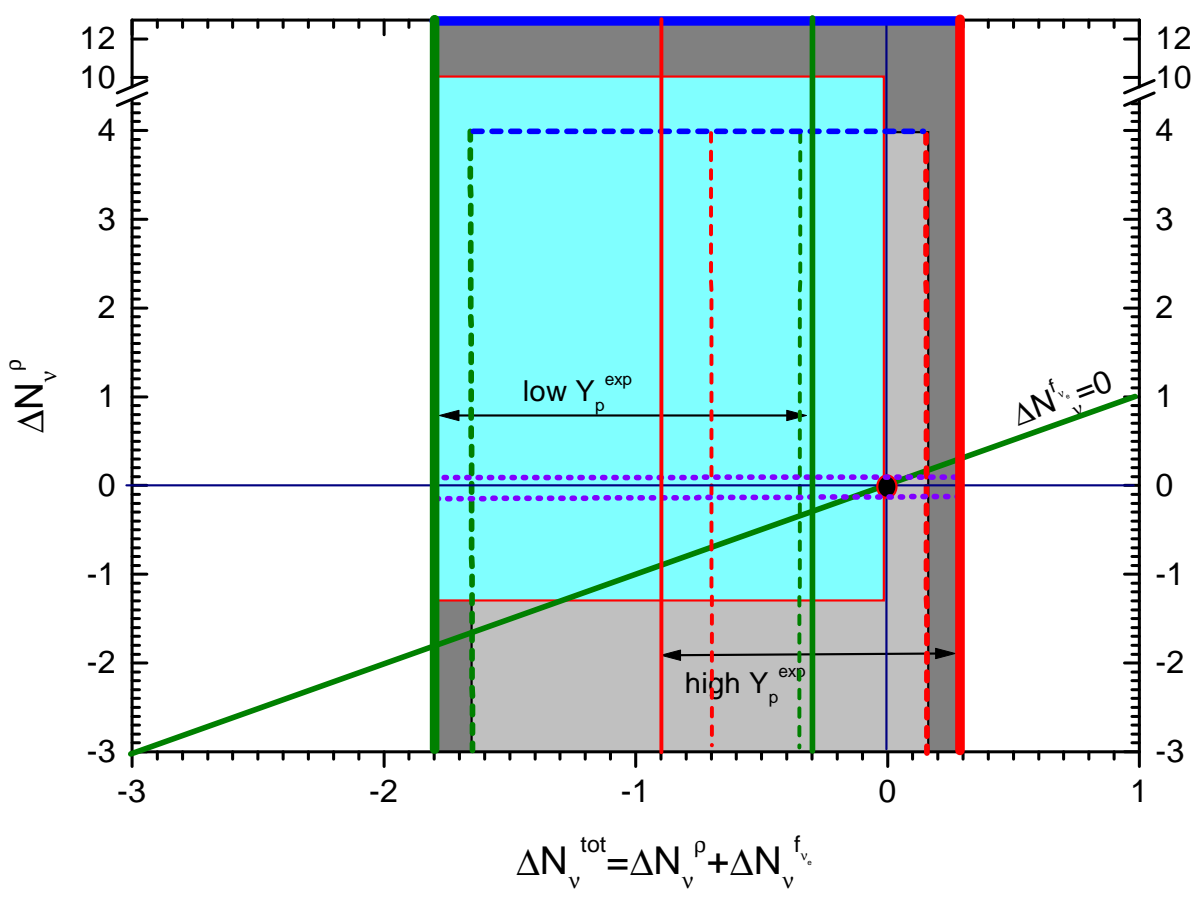


figure2

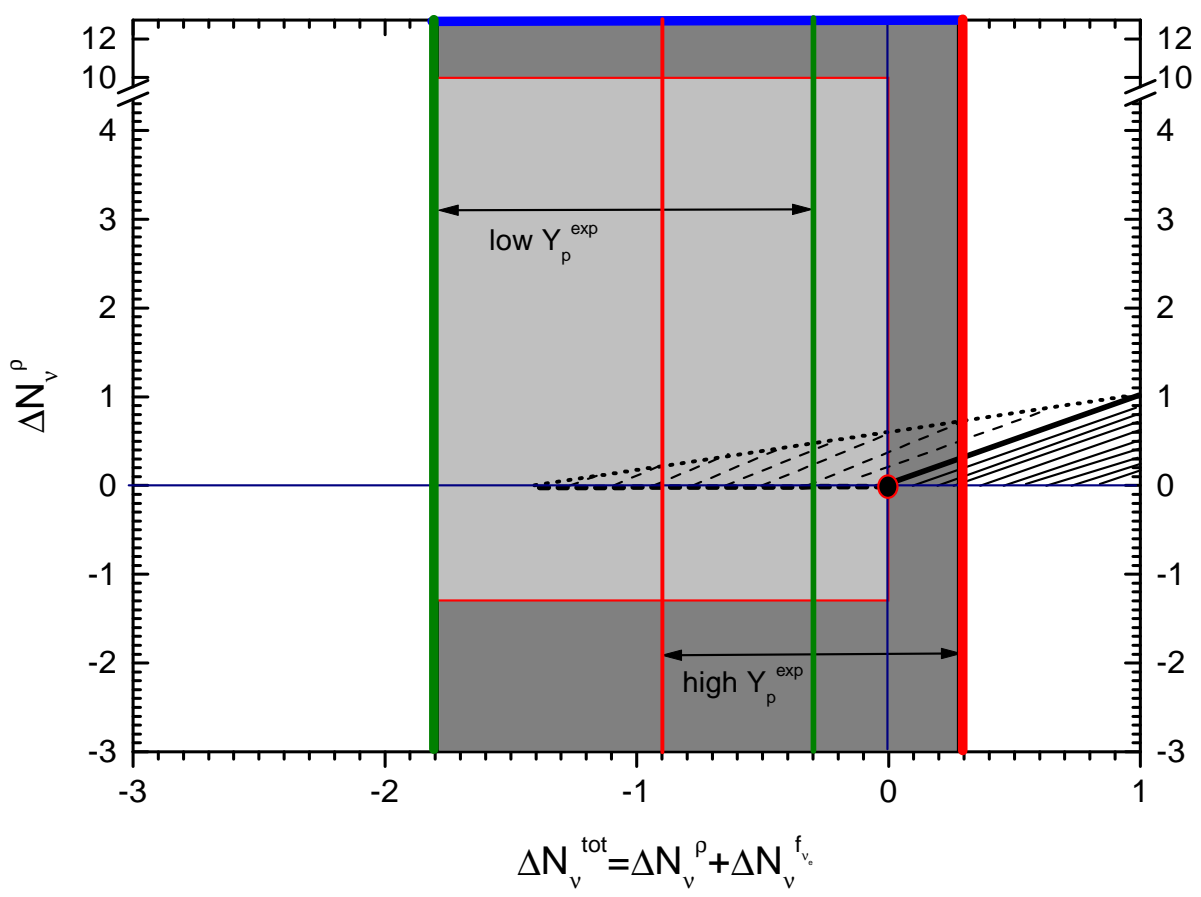


figure3

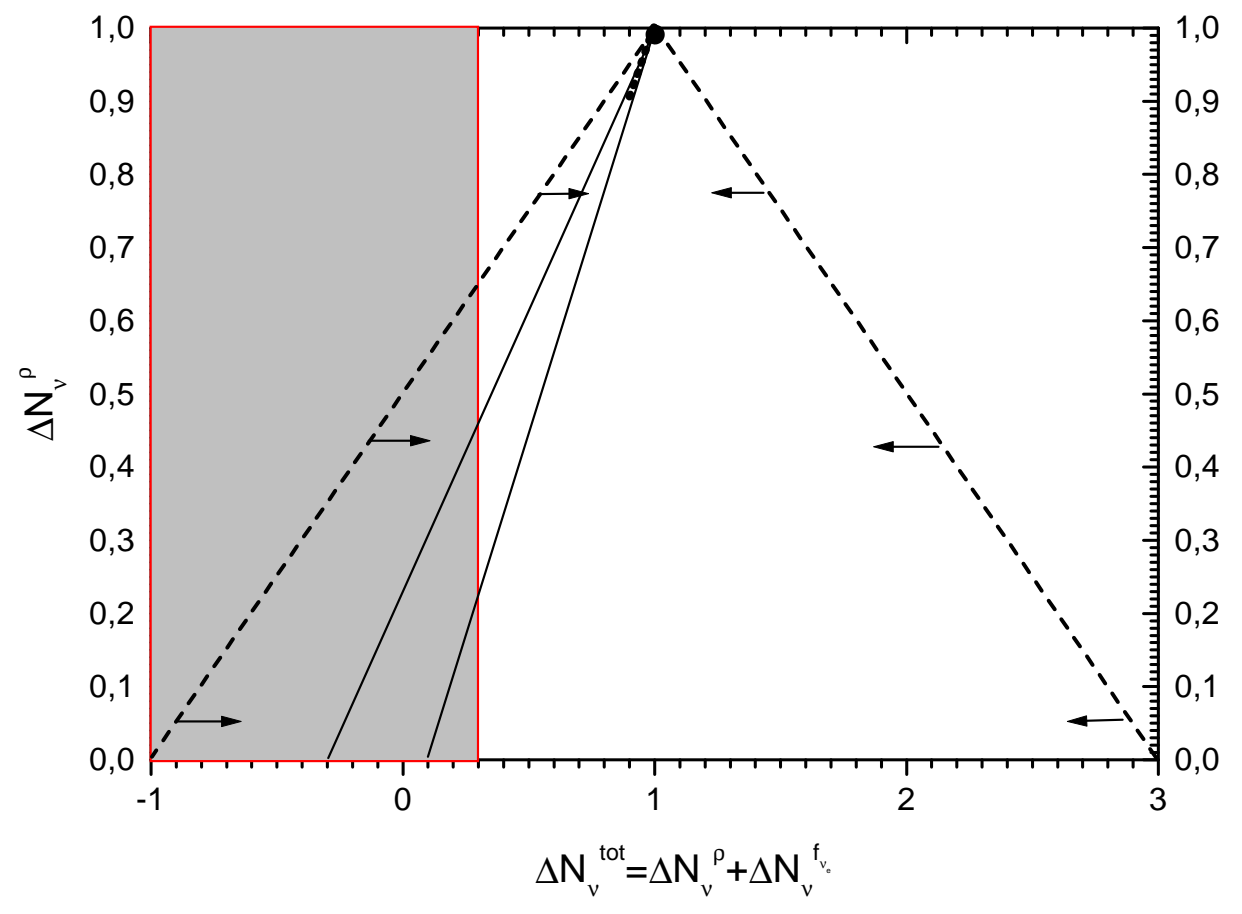

